\title{
Time-course and dynamics of obesity-related behavioral changes induced by energy-dense foods in mice
}

\author{
Jose Espinosa-Carrasco $^{\text {a,b,c** }}$, Aurelijus Burokas ${ }^{\text {d* }}$, Marta Fructuoso ${ }^{a, c}$, Ionas Erb ${ }^{b, c}$, \\ Elena Martín-García ${ }^{d}$, Miriam Gutiérrez-Martos ${ }^{d}$, Cedric Notredame ${ }^{\mathrm{b}, c}$, Rafael \\ Maldonado ${ }^{\mathrm{d}}$, Mara Dierssen ${ }^{\mathrm{a}, \mathrm{c}, \mathrm{e}}$
}

${ }^{a}$ Cellular \& Systems Neurobiology, Systems Biology Program, The Barcelona Institute of Science and Technology, Centre for Genomic Regulation (CRG), Dr. Aiguader 88, 08003 Barcelona, Spain

${ }^{\mathrm{b}}$ Comparative Bioinformatics, Bioinformatics and Genomics Program, Barcelona Institute of Science and Technology, Centre for Genomic Regulation (CRG), Dr. Aiguader 88, 08003 Barcelona, Spain

'Universitat Pompeu Fabra (UPF), Barcelona, Spain

${ }^{\mathrm{d}}$ Laboratori de Neurofarmacologia. Departament de Ciencies Experimentals i de la Salut, Universitat Pompeu Fabra, PRBB, Dr. Aiguader 88, 08003 Barcelona, Spain.

eentro de Investigación Biomédica en Red de Enfermedades Raras (CIBERER), Spain

* These authors contributed equally to this work.

Correspondence should be addressed to: Mara Dierssen. E-mail: $\underline{\text { mara.dierssen@crg.eu }}$

Phone: +34-93-3160140

Fax: +34-93-3160099

Category of the article: Regular research article

Number of references: 50

Words in Abstract: 184

Words in Article (excluding abstract, acknowledgements, references and figure legends): 4648

Number of figures: 7 
Running title: Dynamics of obesity-related behavioral changes

\section{Abstract}

Obesity represents an important risk factor contributing to the global burden of disease. The current obesogenic environment with easy access to calorie-dense foods is fueling this obesity epidemic. However, how these foods contribute to the progression of feeding behavior changes that lead to overeating is not well understood and needs systematic assessment. Using novel automated methods for the high-throughput screening of behavior, we here examine mice meal pattern upon long-term exposure to two types of palatable foods: high-fat chow and a chocolate-mixture. We identified rapid diet-specific behavioral changes after exposure to those high-caloric diets. Mice fed with high-fat chow, showed long-lasting meal pattern disturbances, which initiate with a stable loss of circadian feeding rhythmicity. Mice receiving a chocolatemixture showed qualitatively similar changes, though less marked, disruptions in feeding behavior and the disruption of the circadian feeding rhythmicity was only transient. Strikingly, compulsive-like eating behavior is triggered immediately after exposure to both high-fat food and chocolate-mixture diet, well before any changes in body weight could be observed. We propose these early changes as behavioral biomarkers of prodromal states of obesity that could allow early intervention. 


\section{Introduction}

Obesity is a multifactorial disease whose prevalence has steadily increased to pandemic proportions in the last decades (http://www.who.int, Withrow \& Alter, 2011; Peeters \& Backholer, 2017). Environmental factors, such as exposure to high palatable and high-caloric diets play a key role in the development of overweight and obesity (Hill, 1998; Schröder et al., 2004), whereas genetic variants have limited effects (Naukkarinen et al., 2010; Willyard, 2014). Obesity ultimately results from long-term energy imbalance (Morton et al., 2014), with energy intake exceeding energy outcomes. Besides leading to weight gain and obesity development, excessive consumption of diets laden in saturated fat and refined sugars evoke compulsive behaviors, such as binge eating, food seeking and food craving, as reported in experimental studies with rodents (Corwin and Buda-Levin, 2004; Teegarden and Bale, 2007; Hammerslag and Gulley, 2014).

In animal models, intermittent access to highly palatable and hypercaloric food provokes overconsumption and leads to compulsive-like eating behavior (Avena et al., 2008; Oswald et al., 2011; Ziauddeen and Fletcher, 2013). However, even though longitudinal cohort studies in humans suggest that aberrant eating patterns play a major role in obesity development (Nicklas et al., 2001), the continuous exposure to palatable hypercaloric food has been much less explored, and the existing studies are mainly devoted to the metabolic analysis, and not to the fine-grained behavioral characterization (Sampey et al., 2009; Surwit et al., 1995).

We here performed a longitudinal analysis of the feeding behavior changes induced by highly palatable sugar and fat-laden foods (Yanovski, 2003; Malik et al 2013). For this purpose, we applied novel automated methods for the high-throughput screening of feeding behavior. Specifically, C57BL/6J mice fed with either a high-fat chow or free access to a chocolate-based diet with refined sugars were continuously recorded. The first corresponds to a previously 
validated diet-induced obesity (DIO) model (Hariri and Thibault, 2010; Wang and Liao, 2012) which allowed us to observe feeding behavioral phenotypes in obese animals.

In the second condition, we aimed to reproduce human obesogenic environments where high-dense foods have become increasingly available. Varied, energetically dense diets, also known as "cafeteria" diets have been shown to induce hyperphagia and obesity in rodents. This diet refers to the offering of a choice of high energy/sugar foodstuffs where animals choose what they wish to eat, and thus, it more accurately reflects the variety of highly palatable, energy-dense foods accessible in Western societies and associated with snacking and weight gain (Speakman et al., 2007). The rationale here was to compare the resulting longitudinal phenotypes (meal pattern, circadian rhythms and development of compulsive-like behaviors) 1/ in a condition in which obesity has been induced by a validated model of obesity using a hypercaloric diet alone; 2 / in a condition similar to Western society lifestyle (free choice access between standard chow and chocolate mixture) and 3/ to compare them with respect their controls, a non-obese group having access to a balanced diet (standard chow). Using novel computational meal pattern analysis methods, we found diet-specific patterns of feeding behavior and circadian feeding rhythmicity changes. 


\section{Material and methods}

\section{Animals}

Twelve-week-old C57BL/6J (Charles River, L’Arbresle, France) male mice, weighing 22$28 \mathrm{~g}$ at the beginning of the experiments were used in this study. Mice were individually housed in controlled laboratory conditions (temperature $21 \pm 1^{\circ} \mathrm{C}$; humidity $55 \pm 10 \%$ ) on a 12 hours light/dark cycle (lights off at 20:00 hours). Food and water were available ad libitum except during the sessions of limited access to high-fat or chocolate-mixture. All animals were weighed every 48 hours and cages were deep cleaned every 2 weeks to minimize stress. All experimental protocols were performed in accordance with recommendations for the proper care and use of laboratory animals [local (law 32/2007); European (EU directive $n^{\circ}$ 86/609, EU decree 2001-486) regulations, and the Standards for Use of Laboratory Animals n A5388$01(\mathrm{NIH})$ ] and were approved by the local ethical committee (CEEA-PRBB).

\section{Diets}

We used high-fat chow (experiment 1) or a mixture of commercial chocolates (experiment 2). In the case of experiment 2, standard chow was also available, since in preliminary experiments exclusive chocolate-based diet provoked weight loss and intestinal problems to the mice (data not shown). Mice were randomly assigned to each diet (high-fat or chocolatemixture) or to the control group, with ad libitum access to standard chow (Trans 23 diet, Mucedola, Italy) providing $3.87 \mathrm{kcal} / \mathrm{g}$ (75\% energy from carbohydrates, 18\% from protein and $7 \%$ from fat) and water.

In experiment 1 (high-fat chow), a total of 17 animals were randomly assigned to the control group receiving standard chow $(n=9)$ or to the group that received high-fat chow $(n=8)$, from now on referred as HF group. High-fat chow provides $5.21 \mathrm{kcal} / \mathrm{g}$ (Test Diet ${ }^{\circledR}$, USA; 21\% energy from carbohydrates, $19 \%$ from protein and $60 \%$ from fat). 
In experiment 2 (chocolate-mixture chow), a total of 16 animals were randomly assigned to the control group receiving standard chow $(n=9)$ or to the group that had free choice access to standard chow and to a chocolate mixture $(n=7)$, from now on referred as CM group. The chocolate mixture was manufactured as previously described (Martín-García et al., 2010) using a mix of equal amounts of Bounty ${ }^{\circledR}$, Snickers ${ }^{\circledR}$, Mars ${ }^{\circledR}$ and Milka ${ }^{\circledR}$ chocolates, cut into equal pellets suitable for feeding. Chocolate-mixture chow provided $4.92 \mathrm{kcal} / \mathrm{g}$ (52\% energy from carbohydrates, $17 \%$ from protein and $24 \%$ from fat). All mice had ad libitum access to water.

\section{Food, drink and activity monitoring system}

Animals were individually housed in PheCOMP multi-take metabolism and activity cages (Panlab-Harvard Instruments, Barcelona, Spain). For a detailed description of this highresolution monitoring system see (Bura et al., 2010). Meal pattern analysis included calculations of time courses of food/drink access, duration and recurrence intervals of eating or drinking acts, circadian and ultradian periodicities, and event-related frequency distributions. Animals were recorded uninterruptedly during periods of 3 or 4 days. Between these periods the system was paused during approximately one hour, for refilling the feeders and the cleaning of the cages, during which animals had no access to the food.

Feeding/drinking acts were considered as a meal or a drinking bout when separated by more than 120 seconds from the next feeding/drinking act. Hence, feeding/drinking acts were merged into a single food/water bout when the intermeal interval was shorter than 120 seconds (see Heyne et al 2009).

\section{Experimental design}


The experiments were divided in three phases (Fig. 1): a one-week habituation phase previous to the introduction of the craved foods, an obesity development phase of 8 weeks, in which animals developed overweight, and finally a behavioral testing phase.

Habituation phase. All mice were individually housed in PheCOMP cages and provided with standard chow and water ad libitum during one week to allow habituation to the housing conditions and to define the baseline meal pattern (habituation phase; Fig. 1).

Obesity development phase. After habituation, each group of animals was offered their corresponding diet, i.e. high-fat chow (experiment 1) or chocolate-mixture and standard chow (experiment 2). Control groups of both experiments continued receiving access to standard chow. The development of overweight in the experimental groups was followed during 8 weeks (obesity development phase; Fig. 1).

Testing phase. At the end of the obesity development phase, we analyzed the changes in meal pattern upon limited access to high-fat food or chocolate-mixture, respectively, as previously described (Heyne et al., 2009). Briefly, access to high-fat or chocolate-mixture diet was restricted to 1 hour during the light (resting) phase of the light-dark cycle for 3 consecutive days in overweight mice. Standard chow and water were provided ad libitum. This limited access allows evaluating behavioral flexibility, defined as the ability of the individual to adapt to a new feeding situation. To compensate the restriction of access to the high-caloric food, flexible animals will increase the intake from the available energy source (standard chow). In CM mice, we compared energy intake and meal size of high-fat or chocolate-mixture during restriction to freely available conditions. In HF animals, since they did not receive standard chow in pre-test conditions, we compared only the energy intake of high-fat food during the 23 hours in basal condition with the energy intake of standard chow during the 23 hours of the test. This procedure allowed to evaluate if the animals compensate for the restriction of access 
to the high caloric food by increasing the intake of the low caloric food. An inflexible response would be revealed by the neglect of standard chow.

\section{Data analysis}

All results are expressed as mean \pm S.E.M. Data were collected using PheCOMP multi-take metabolism and activity cages (Panlab-Harvard Instruments, Barcelona, Spain) and were further processed using Perl and R scripts (R Core Team, 2015). In this manner, we improved the longitudinal dimension of the data concatenating all the recordings as Compulse ${ }^{\circledR}$, the software provided by the manufacturer, allows only file-by-file analysis. Data concatenation and self-analysis allowed graphical data representations, providing a better framework for data handling and interpretation. Fold change significances were computed using the Wilcoxon rank-sum test.

Individual group comparisons within experiments were performed using one-way analysis of variance (ANOVA). Differences during limited access to food and basal conditions were calculated using one-way repeated-measures ANOVA. Average intake values during the development phase were analyzed using three-way ANOVA with diet as between-subjects factor (high-fat/standard chow, chocolate-mixture/standard chow) and phase (dark/light) together with time (development phase) as within-subjects factor. Data from short deprivation periods for cleaning the cages were analyzed by two-way repeated measures ANOVA with diet (high-fat/standard chow) as between subjects factor and time (24 hours before and 24 hours after deprivation) as within-subjects factor. Post-hoc analyses were performed when required (Bonferroni). No statistical comparisons were run between the two diet groups since the two experiments were not carried out simultaneously. Each of the experiments had a control group to control for any posible batch effect. The statistical analysis was performed using SPSS 
software version 17.0 (SPSS Inc., Chicago, IL, USA). Only P-values under 0.05 were considered significant.

\section{Results}

\section{Mice exposed to high-fat and chocolate-mixture showed diet-specific meal pattern modifications}

To investigate diet-specific behavioral changes, we analyzed meal pattern differences of mice exposed to a high-fat or to a chocolate-mixture diet. These experiments were performed at the end of the obesity development phase (Fig. 1), once the mice had already reached a stable overweight.

Mice exposed to high-fat chow showed a significant increase of body weight with respect to their basal values, which was $40 \%$ higher than in the standard chow group (one-way ANOVA; $F_{(1,15)}=65.632 ; P<0.001$, Fig. 2 A left $)$. This increase was associated to a significant increase of energy intake (one-way ANOVA; $F_{(1,15)}=57.91 ; P<0.001$, Fig. $2 \mathrm{~B}$ left) and of the total amount of food consumed (one-way ANOVA; $F_{(1,15)}=22.191 ; \mathrm{P}<0.001$, Fig. 2C left). We also detected increased number of meals (one-way ANOVA; $F_{(1,15)}=13.804 ; \mathrm{P}<0.01$, Fig. $2 \mathrm{D}$ left) of shorter duration than those of the standard chow group (one-way ANOVA; $F_{(1,15)}=$ 6.662; $\mathrm{P}<0.01$, Fig. 2E left), revealing a higher eating rate, i.e. the food consumed per time unit (mg/sec) (one-way ANOVA; $F_{(1,15)}=154.17 ; P<0.001$, Fig. $2 \mathrm{~F}$ left). Finally, satiety ratio, defined by the time elapsed until re-feeding $(\mathrm{min} / \mathrm{KJ})$, was significantly reduced in $\mathrm{HF}$ mice (one-way ANOVA; $F_{(1,15)}=15.47 ; P<0.01$, Fig. $2 \mathrm{G}$ left), indicating that even though high-fat chow provides more energy, mice are less satiated and re-feed more frequently. 
Mice with free access to chocolate-mixture also showed a significant $10 \%$ higher weight gain compared to their control group (one-way ANOVA; $F_{(1,14)}=5.73 ; P<0.05$, Fig. $2 \mathrm{~A}$ right), though much less important than in HF mice. Also, the increase in energy intake in CM animals (one-way ANOVA; $F_{(1,14)}=20.62 ; P<0.001$, Fig. 2B right), was less important than in HF mice (HF group: 6665 \pm 679.4 kJ/kg; CM group: $2500 \pm 294.6 \mathrm{~kJ} / \mathrm{kg}$ ), and mainly due to the chocolate-mixture (one-way ANOVA: $F_{(1,12)}=6.34 ; P<0.05$, inset of Fig. 2B right), while the total amount of food consumed by CM mice was not significantly higher than total food intake of the control group (one-way ANOVA; $F_{(1,14)}=0.704$; n.s., Fig. 2C right). CM mice consumed a similar amount of chocolate-mixture than of standard chow (one-way ANOVA; $F_{(1,12)}=1.38$; n.s., inset of Fig. 2C right), suggesting no preference for chocolate. Even so, as in the HF mice, the CM group showed a significant increase of total number of meals (oneway ANOVA; $F_{(1,14)}=8.18 ; P<0.05$, Fig. $2 \mathrm{D}$ right), of significantly reduced duration (oneway ANOVA; $F_{(1,14)}=7.68 ; P<0.05$, Fig. 2 E right), due to the shorter duration of chocolatemixture meals compared with standard chow meals (one-way ANOVA; $F_{(1,12)}=211.47 ; P<$ 0.001, inset of Fig. 2E right). Similar to HF animals, the eating rate of CM mice was higher in comparison to control mice (one-way ANOVA; $F_{(1,14)}=22.78 ; P<0.001$, Fig. $2 \mathrm{~F}$ ) due to an increased eating rate of the chocolate-mixture (one-way ANOVA; $F_{(1,12)}=3.554, P<0.001$, inset of Fig. 2F). Finally, CM mice as opposed to HF mice, increased the overall satiety ratio (one-way ANOVA; $F_{(1,14)}=7.68 ; P<0.05$, Fig. $2 \mathrm{G}$ ), mainly due to the increased satiety for the standard chow in this group (CM group, standard chow: 72,52 $\pm 8.8 \mathrm{~min} / \mathrm{kJ}$; control group, standard chow: $33.94 \pm 1.34 \mathrm{~min} / \mathrm{kJ}$ ). Although, CM mice showed a tendency to reduce satiety ratio for chocolate-mixture (one-way ANOVA; $F_{(1,12)}=2.72$, n.s., inset of Fig. 2G).

Neither HF nor CM mice showed significant changes in locomotor activity with respect to their controls (data not shown). 


\section{Longitudinal behavioral analysis revealed early disruption of meal patterns in HF and}

\section{CM mice}

To grasp the whole significance of behavioral repertoires, we performed thorough time series analyses that would reveal more details about the behavioral trajectories. To identify all the discrete meal pattern changes along a time course in obesogenic environments, main obesityrelated behavioral changes were segmented, quantifying them per week. Figures 3 and 4 represent how behaviors unfold on different time scales and their hierarchical relationships. As a control, we also quantified drinking behavior.

During the habituation phase, in which both HF and CM groups received ad libitum standard chow, no differences in feeding behavior were detected with respect to their controls (Figs. 3A and 4A), indicating a basal feeding pattern that was similar in all mice. In the HF group, meal pattern disruptions unfold on a short time scale and display low variability. The first week upon exposure to high-fat, we already detected significant increases in food intake, number of meals, and eating rate with respect to the control group, while the duration of each meal was reduced (Fig. 3B). These results indicate a rapid development of hyperphagia and disruption of feeding behavior. Besides, the average intermeal duration was reduced (Fig. 3B), revealing an early disruption of homeostatic satiety signals. Regarding drinking behavior only slight changes were detected in the HF group, consisting in a reduction of drinking bouts and increased interbout interval (Fig. 3).

In CM mice we analyzed separately the feeding behaviors related to chocolate-mixture and standard chow (Fig. 4B, C and Fig. S1). In this group, the changes were less marked than in the HF group and were different in the initial period of chocolate exposure than in the late period when mice are already overweighed. In the first weeks of exposure, mice showed a clear 
preference for chocolate-mixture chow, with increased intake of chocolate-mixture and reduced amount of standard chow consumed per meal (Fig. 4B and C). Interestingly, chocolatemixture meals were significantly shorter (Fig. 4C) than standard chow meals (Fig. S1), the eating rate for chocolate-mixture significantly and steadily increased with respect to standard chow. We also found an increase in the intermeal duration, greater for standard chow than for chocolate-mixture, suggesting that in this case satiety is preserved. Remarkably, each behavioral feature also had a rich dynamical profile with differential properties and patterns of oscillations. While intake of chocolate-mixture was only increased during the first three weeks of access, the increase of eating rate and shortening of meal duration were significant during the whole obesity development period, indicating that the temporal scale at which different behaviors unfold differs. No major differences were detected in drinking behavior of CM mice.

\section{Early obesity-related circadian feeding pattern alteration upon introduction of energy dense diets}

Individual differences in behavior and their underlying mechanisms are an increasingly relevant research field. Behavioral readouts present a high degree of intraindividual and interindividual variability. Individual behavioral tendencies can be temporally consistent, meaning that the individual's traits stay similar across time, or present variability even on short temporal scales due to specific processes such as habituation, or sensitization. To characterize the development of meal pattern disturbances leading to overeating in individual mice, we chronically analyzed the fine-grained pattern of feeding changes upon introduction of energydense diets. In mice, feeding is a patterned activity subject to well-controlled daily oscillations. As such, during the habituation phase before exposure to high-fat food or chocolate-mixture, feeding behavior showed a circadian organization in all groups of mice, with feeding bouts accumulating over the dark (active) phase of the light/dark cycle (nocturnal meals) and being 
almost absent during the light (resting) phase (HF mice depicted in Fig. 5A and CM mice in Fig. 6A), indicating a stable feeding pattern along time that was similar in all groups.

As depicted in Fig. 5B, immediately after introduction of the high-fat chow, mice increased food intake and lost the circadian feeding rhythmicity as shown by an increase of feeding events in the light phase. This phenotypic change was detected in all but one HF mouse already the first days after exposure to high-fat chow. Also, our longitudinal analysis revealed that the pattern of the behavioral changes is slightly different in each individual.

Even so, the amount of food consumed during light and dark phases by HF animals was significantly higher than by control mice during light and dark periods (three-way ANOVA: $F_{(1,15)}=199.57 ; P<0.001$, Fig. S2A). Also, there was a clear difference in the amount of food consumed during day and night (three-way ANOVA: $F_{(1,15)}=97.31 ; P<0.01$, Fig. S2A) and a significant interaction of diet, phase and days of exposure to high-fat (three-way repeated measures ANOVA; $F_{(7,105}=4.12 ; P<0.001$, Fig. S2A). HF animals dramatically increased food consumption during both light and dark periods in comparison to control mice, which consumed low amounts of food during the light phase (Control light phase vs. HF light phase: Bonferroni post-hoc analysis, $P<0.001$; Control dark phase vs. HF dark phase: Bonferroni post-hoc analysis, $P<0.001)$.

Interestingly, in the HF group, our longitudinal recordings allowed to identify that short food deprivation periods as those occurring when maintenance cleaning was performed (approximately one hour; 'cleaning deprivation') caused a binge-like eating behavior in HF animals (Fig. 5C). To quantify this observation, we compared the food consumed during 30 minutes after cleaning to the same period of previous and following days. Two-way repeatedmeasures ANOVA revealed a significant effect of diet $\left(F_{(1,15)}=91.25 ; P<0.001\right.$, Fig. S2B), time $\left(F_{(2,30)}=62.512 ; P<0.001\right.$, Fig. S2B $)$ and an interaction of diet and time $\left(F_{(2,30)}=59.26\right.$; 
$P<0.001$, Fig. S2B). The high-fat binges after 'cleaning deprivation', were significant compared to high-fat consumption the previous or following day (high-fat consumption previous day vs. high-fat after deprivation: Bonferroni post-hoc, $P<0.001$; high-fat following day vs. high-fat after deprivation: Bonferroni post-hoc, $P<0.001)$.

To determine the extent of this phenotype, in a separate one-day experiment after mice were already overweight, we limited high-fat access to one hour, while mice received standard chow the rest of the day. During the one-hour access to high-fat chow, HF mice showed significantly increased of energy intake (One-way repeated measures ANOVA; $F_{(1,15)}=5.733 ; P<0.05$, Fig. 7A), meal size (One-way repeated measures ANOVA; $F_{(1,15)}=7.630 ; P<0.05$, Fig. 7B) and eating rate (One-way repeated measures ANOVA; $F_{(1,15)}=7.037: P<0.05$, Fig. 7C), compared to the same period before limited access. Remarkably, the energy intake of standard chow during the limited access experiment was very low and highly reduced when compared with the energy intake having as source high-fat chow in basal conditions (one-way repeated measures ANOVA; $F_{(1,15)}=84.552 ; P<0.001$, Fig. 7A inset). This reduction suggests that mice "wait” for their preferred food instead of compensating the caloric intake.

In the CM group, after the introduction of the chocolate-mixture (Fig. 6B), animals also showed some feeding bursts during the light (inactive) period, but the circadian feeding rhythmicity was not significantly affected. This disruption of feeding behavior mainly affected the eating pattern of the chocolate-mixture, while standard chow meals remained unaltered (Fig. 6C). We could also observe a higher inter-individual variability in this group, and no “cleaning starvation” episodes were detected.

In the limited access experiment to chocolate-mixture, mice significantly increased chocolate-mixture energy intake (one-way repeated measures ANOVA; $F_{(1,12}=5.716$; $P<$ 0.05, Fig. 7D) and meal size (one-way repeated measures ANOVA; $F_{(1,12}=5.56 ; P<0.05$, Fig. 
7F) upon re-offer, whereas eating rate did not reach significance (one-way repeated measures ANOVA; $F_{(1,12}=0.98$; n.s., Fig. 7E). Interestingly, $\mathrm{CM}$ animals did not significantly increase standard chow energy intake to compensate the energy unbalance during the 23 remaining hours of the test during which mice have only access to standard chow (one-way repeated measures ANOVA; $F_{(1,6)}=2.08$; n.s, Fig. 7D, inset). 


\section{Discussion}

Using a high-throughput automated recording system, and a novel analysis framework, we identified differential changes in feeding behavior promoted by a high-fat food with low carbohydrate content and a chocolate-based food with high carbohydrate content (chocolatemixture) at the initial phases of obesity development. Our results suggest diet-specific almost immediate increased food intake and eating rate, along with a reduced meal duration and intermeal intervals as compared to standard chow, leading to an overeating phenotype. We detected a loss in circadian feeding rhythmicity, which was more marked upon high-fat diet, and inter-individual differences in the response to these diets. This information is key to understanding how different types of high-energy diets influence obesity's natural history.

Almost immediately upon exposure to high-fat or chocolate-mixture food, both diets reduced meal duration, suggesting higher intrameal satiety for the energy-dense diets than for standard chow. One of the homeostatic reasons behind intrameal satiety is the adaptation of the energy amount per meal to the individual requirements (Benelam, 2009). However, the caloric intake in the HF and CM groups was higher than in controls, in accordance to previous work showing that high-fat diet reduces response to satiation signals when compared with a similar caloric intake high-carbohydrate diet (Nefti et al., 2009). This reduction can further explain the differences on intrameal satiation between the high-fat and the chocolate-mixture food, since fat content is lower in the latter. Indeed, fat produces a potent oral stimulation that increases the intake of food consumed before satiation signals become effective, known as the fat paradox (Blundell \& Macdiarmid, 1997). Thus, in spite of its high energy content, fat food produces a delay of the satiation signals, which provokes its passive overconsumption and consequently an increase of the energy consumption, as reported in our study.

Intermeal satiety, the second component that determines the total energy intake of individuals, was also modified in both groups. The satiety state determines the delay of next 
meal onset and reduces food consumption at the next eating occasion (Gerstein et al., 2004). Given the high-energy content of both high-fat and chocolate-mixture foods, a high satiety promoted by these foods would be expected as a homeostatic response. However, compared to control mice, HF mice presented the lowest satiety ratio while the CM mice increased their global satiety rate, but showed lower satiety for chocolate-mixture than for standard chow. Accordingly, time between meals was reduced confirming that postprandial satiety is lower in food rich in fat than in food rich in carbohydrates (Mattes et al., 2005). The deficient homeostatic response can be explained by the energy density and/or the high palatability of the craved foods (Gerstein et al., 2004). Our results support that the disruption of homeostatic control of eating is mainly due to food fat content, regardless the palatability of the food.

In our experiment, both HF and CM mice showed compulsive-like eating behavior (Cottone et al., 2012) a factor behind some kinds of obesity (Heyne et al., 2009). Precisely upon obesity development, the HF group showed the highest eating rate while CM mice presented enhanced eating rate for the hopper containing chocolate-mixture. This difference may be partially explained by the effect of fat oral stimulation, which facilitates its ingestion and its abovementioned overconsumption. However, increased eating rate could also reflect compulsivelike feeding behavior. Supporting this view, limited access to high-fat chow produced changes in feeding behavior during the access period similar to those described in binge-eating models (Bake et al., 2014; Berner et al., 2008). To our knowledge, among carbohydrates only sugar has been shown to promote binge eating (Avena, 2010). We therefore hypothesized that the sugar content of our chocolate-mixture diet (44\%) might not be enough to trigger the observed response. Neither diet did compensate the energy intake by increasing standard chow consumption during limited access. We suggest that mice suppressed the consumption of standard chow because they expected a more preferred substance as an anticipatory negative contrast response (Cottone et al., 2008), denoting food-taking inflexibility. 
One of the most interesting findings in our work is that mice altered their feeding behavioral patterns immediately after the introduction of the energy-dense diets. First, compulsive-like feeding and specifically binge-like eating (i.e. large, short-duration meals) appeared immediately after exposition to high-fat food well before obesity had developed, as evidenced by the burst of high-fat food consumption following the short deprivation periods due to cage cleaning. Compulsive-like eating behavior has been described after limiting food access (Avena, 2010; Cottone et al., 2012). This so-called deprivation effect defines an increased intake for a substance after an abstinence period and is used as a marker of craving (Avena et al., 2005; Khisti et al., 2006). Such behavior was shown upon intermittent access to sugar after a two weeks deprivation, and after withdrawal of chronic high-fat access (Sharma et al., 2013), but all the studies have assumed that this is a long-term phenomenon. Our longitudinal data visualization and analysis allowed the detection of compulsivity towards high-fat food already after three days of ad libitum exposure to high fat. Free access to chocolate-mixture did not promote this binge-like eating behavior, but other markers of compulsive-like feeding behavior such as eating rate were early increased in CM mice.

As compared to standard chow, both diets early disrupted circadian feeding organization, although more remarkably in the case of high-fat diet. Previous studies demonstrated a disruption of circadian molecular and behavioral rhythm after several weeks (Hatori et al., 2012; Kohsaka et al., 2007), or immediately after high-fat exposure (Pendergast et al., 2013). Nonetheless, to our knowledge there are no studies reporting circadian rhythm alterations triggered by ad libitum intake of palatable diets like in the chocolate-mixture condition. Interestingly, the circadian rhythm of CM mice was only disrupted during the first three weeks, a period that corresponded with the deepest feeding pattern alterations, suggesting that changes triggered by chocolate-mixture were only transient and observable in early stages after the introduction of the chocolate-mixture diet. 
The classical view supports that a high-fat diet is largely responsible for weight gain while sugar is responsible for addictive-like behavior. We here show that both high fat and a carbohydrate rich (chocolate-mixture) chow are able to modify the baseline feeding behavior of mice maintained in balanced diet conditions. However, our results also suggest that a forced high-fat content might be a stronger factor than sugar content triggering both motivational reward and entraining circadian oscillators. Together both sides of the coin might play a main role in the boosting of abnormal eating behaviors. As shown here, the study of the time course of obesity-related behaviors using high-resolution, long-term recording of data might provide insights into the natural history of obesity, leading to detection of prodromal behavioral markers. 


\section{Acknowledgements}

The laboratory of M.D. is supported by DIUE de la Generalitat de Catalunya (Grups consolidats SGR 2014/1125). This work was supported by Fondation Jérôme Lejeune (Paris, France), MINECO (SAF2013-49129-C2-1-R), CDTI (“Smartfoods”) and EU (Era Net Neuron PCIN-2013-060). The CRG is a Center of Excellence Severo Ochoa SEV-2012-0208. The CIBER of Rare Diseases is an initiative of the ISCIII.

The laboratory of C.N. acknowledge the funding from the Spanish Ministry of Economy and Finance (MINECO), grant number BFU2011-28575. J.E. received the FI grant from Agència de Gestió d'Ajuts Universitaris i de Recerca (AGAUR) de la Generalitat de Catalunya.

This work was supported by the "Ministerio de Economía y Competitividad-MINECO" (SAF2011-29846 and \#SAF2014-59648-P), the “Instituto de Salud Carlos III” (RETICS-RTA, \#RD12/0028/0023), the "Generalitat de Catalunya-AGAUR” (\#2014-SGR-1547) and the European Commission (NeuroPain, \#HEALTH-F2-2013-602891) to R.M.

The authors declare no competing financial interests. 


\section{References}

Avena, N.M., 2010. The study of food addiction using animal models of binge eating. Appetite 55, 734-7. doi:10.1016/j.appet.2010.09.010

Avena, N.M., Long, K. a, Hoebel, B.G., 2005. Sugar-dependent rats show enhanced responding for sugar after abstinence: evidence of a sugar deprivation effect. Physiol. Behav. 84, 359-62. doi:10.1016/j.physbeh.2004.12.016

Avena, N.M., Rada, P., Hoebel, B.G., 2008. Evidence for sugar addiction: behavioral and neurochemical effects of intermittent, excessive sugar intake. Neurosci. Biobehav. Rev. 32, 20-39. doi:10.1016/j.neubiorev.2007.04.019

Bake, T., Morgan, D.G. a, Mercer, J.G., 2014. Feeding and metabolic consequences of scheduled consumption of large, binge-type meals of high fat diet in the SpragueDawley rat. Physiol. Behav. 128, 70-9. doi:10.1016/j.physbeh.2014.01.018

Benelam, B., 2009. Satiation, satiety and their effects on eating behaviour. Nutr. Bull. 34, 126-173. doi:10.1111/j.1467-3010.2009.01753.x

Berner, L. a, Avena, N.M., Hoebel, B.G., 2008. Bingeing, self-restriction, and increased body weight in rats with limited access to a sweet-fat diet. Obesity (Silver Spring). 16, 1998-2002. doi:10.1038/oby.2008.328

Blundell, J.E., Macdiarmid, J.I., 1997. Fat as a Risk Factor for Overconsumption: Satiation, Satiety, and Patterns of Eating. J. Am. Diet. Assoc. 97, S63-S69. doi:http://dx.doi.org/10.1016/S0002-8223(97)00733-5

Bura, S.A., Burokas, A., Martín-García, E., Maldonado, R., 2010. Effects of chronic nicotine on food intake and anxiety-like behaviour in $\mathrm{CB}(1)$ knockout mice. Eur. Neuropsychopharmacol. 20, 369-78. doi:10.1016/j.euroneuro.2010.02.003

Corwin, R.L., Buda-Levin, A., 2004. Behavioral models of binge-type eating. Physiol. Behav. 82, 123-30. doi:10.1016/j.physbeh.2004.04.036

Cottone, P., Sabino, V., Steardo, L., Zorrilla, E.P., 2008. Opioid-dependent anticipatory negative contrast and binge-like eating in rats with limited access to highly preferred food. Neuropsychopharmacology 33, 524-35. doi:10.1038/sj.npp.1301430

Cottone, P., Wang, X., Park, J.W., Valenza, M., Blasio, A., Kwak, J., Iyer, M.R., Steardo, L., Rice, K.C., Hayashi, T., Sabino, V., 2012. Antagonism of sigma-1 receptors blocks compulsive-like eating. Neuropsychopharmacology 37, 2593-604. doi:10.1038/npp.2012.89

Gerstein, D.E., Woodward-Lopez, G., Evans, A.E., Kelsey, K., Drewnowski, A., 2004. Clarifying concepts about macronutrients' effects on satiation and satiety. J. Am. Diet. Assoc. 104, 1151-3. doi:10.1016/j.jada.2004.04.027

Hansen, M.J., Schiöth, H.B., Morris, M.J., 2005. Feeding responses to a melanocortin agonist and antagonist in obesity induced by a palatable high-fat diet. Brain Res. 1039, 137-45. doi:10.1016/j.brainres.2005.01.063 
Hariri, N., Thibault, L., 2010. High-fat diet-induced obesity in animal models. Nutr. Res. Rev. 23, 270-99. doi:10.1017/S0954422410000168

Hatori, M., Vollmers, C., Zarrinpar, A., DiTacchio, L., Bushong, E. a, Gill, S., Leblanc, M., Chaix, A., Joens, M., Fitzpatrick, J. a J., Ellisman, M.H., Panda, S., 2012. Timerestricted feeding without reducing caloric intake prevents metabolic diseases in mice fed a high-fat diet. Cell Metab. 15, 848-60. doi:10.1016/j.cmet.2012.04.019

Heyne, Kiesselbach, C., Sahún, I., McDonald, J., Gaiffi, M., Dierssen, M., Wolffgramm, J., 2009. An animal model of compulsive food-taking behaviour. Addict. Biol. 14, 37383. doi:10.1111/j.1369-1600.2009.00175.x

Hill, J.O., 1998. Environmental Contributions to the Obesity Epidemic. Science (80-. ). 280, 1371-1374. doi:10.1126/science.280.5368.1371

Khisti, R.T., Wolstenholme, J., Shelton, K.L., Miles, M.F., 2006. Characterization of the ethanol-deprivation effect in substrains of C57BL/6 mice. Alcohol 40, 119-26. doi:10.1016/j.alcohol.2006.12.003

Kohsaka, A., Laposky, A.D., Ramsey, K.M., Estrada, C., Joshu, C., Kobayashi, Y., Turek, F.W., Bass, J., 2007. High-fat diet disrupts behavioral and molecular circadian rhythms in mice. Cell Metab. 6, 414-21. doi:10.1016/j.cmet.2007.09.006

Martín-García, E., Burokas, A., Martín, M., Berrendero, F., Rubí, B., Kiesselbach, C., Heyne, A., Gispert, J.D., Millán, O., Maldonado, R., 2010. Central and peripheral consequences of the chronic blockade of CB1 cannabinoid receptor with rimonabant or taranabant. J. Neurochem. 112, 1338-13351. doi:10.1111/j.14714159.2009.06549.x

Mattes, R.D., Hollis, J., Hayes, D., Stunkard, A.J., 2005. Appetite: measurement and manipulation misgivings. J. Am. Diet. Assoc. 105, S87-97. doi:10.1016/j.jada.2005.02.029

Morton, G.J., Meek, T.H., Schwartz, M.W., 2014. Neurobiology of food intake in health and disease. Nat. Publ. Gr. 15, 367-378. doi:10.1038/nrn3745

Naukkarinen, J., Surakka, I., Pietiläinen, K.H., Rissanen, A., Salomaa, V., Ripatti, S., YkiJärvinen, H., van Duijn, C.M., Wichmann, H.-E., Kaprio, J., Taskinen, M.-R., Peltonen, L., 2010. Use of genome-wide expression data to mine the "Gray Zone" of GWA studies leads to novel candidate obesity genes. PLoS Genet. 6, e1000976. doi:10.1371/journal.pgen.1000976

Nefti, W., Chaumontet, C., Fromentin, G., Tome, D., Darcel, N., Darcel, N.A., 2009. A highfat diet attenuates the central response to within-meal satiation signals and modifies the receptor expression of vagal afferents in mice. Am J Physiol Regul Integr Comp Physiol 1681-1686. doi:10.1152/ajpregu.90733.2008.

Nicklas, T.A., Baranowski, T., Cullen, K.W., Berenson, G., 2001. Eating patterns, dietary quality and obesity. J. Am. Coll. Nutr. 20, 599-608. doi:10.1080/07315724.2001.10719064

Oswald, K.D., Murdaugh, D.L., King, V.L., Boggiano, M.M., 2011. Motivation for palatable food despite consequences in an animal model of binge eating. Int. J. Eat. Disord. 44, 
Pendergast, J.S., Branecky, K.L., Yang, W., Ellacott, K.L.J., Niswender, K.D., Yamazaki, S., 2013. High-fat diet acutely affects circadian organisation and eating behavior. Eur. J. Neurosci. 37, 1350-6. doi:10.1111/ejn.12133

R Core Team, 2015. R: A Language and Environment for Statistical Computing.

Sampey, B.P., Vanhoose, A.M., Winfield, H.M., Freemerman, A.J., Muehlbauer, M.J., Fueger, P.T., Newgard, C.B., Makowski, L., 2009. Cafeteria Diet Is a Robust Model of Human Metabolic Syndrome With Liver and Adipose Inflammation : Comparison to HighFat Diet. Obesity 19, 1109-1117. doi:10.1038/oby.2011.18

Schröder, H., Marrugat, J., Vila, J., Covas, M.I., Elosua, R., 2004. Adherence to the traditional mediterranean diet is inversely associated with body mass index and obesity in a spanish population. J. Nutr. 134, 3355-61.

Sharma, S., Fernandes, M.F., Fulton, S., 2013. Adaptations in brain reward circuitry underlie palatable food cravings and anxiety induced by high-fat diet withdrawal. Int. J. Obes. (Lond). 37, 1183-91. doi:10.1038/ijo.2012.197

Speakman, J., Hambly, C., Mitchell, S., Król, E., 2007. Animal models of obesity 8, 55-61.

Surwit, R.S., Feinglos, M.N., Rodin, J., Sutherland, A., Petro, a E., Opara, E.C., Kuhn, C.M., Rebuffé-Scrive, M., 1995. Differential effects of fat and sucrose on the development of obesity and diabetes in C57BL/6J and A/J mice. Metabolism. 44, 645-51.

Teegarden, S.L., Bale, T.L., 2007. Decreases in dietary preference produce increased emotionality and risk for dietary relapse. Biol. Psychiatry 61, 1021-9. doi:10.1016/j.biopsych.2006.09.032

Wang, C.-Y., Liao, J.K., 2012. A Mouse Model of Diet-Induced Obesity and Insulin Resistance. Methods Mol. Biol. 821, 421-433. doi:10.1007/978-1-61779-430-8_27

Willyard, C., 2014. Heritability: The family roots of obesity. Nature 508, S58-S60.

Withrow, D., Alter, D. a, 2011. The economic burden of obesity worldwide: a systematic review of the direct costs of obesity. Obes. Rev. 12, 131-41. doi:10.1111/j.1467789X.2009.00712.x

Yanovski, S., 2003. Sugar and Fat: Cravings and Aversions. J. Nutr. 133, 835S-837.

Ziauddeen, H., Fletcher, P.C., 2013. Is food addiction a valid and useful concept? Obes. Rev. 14, 19-28. doi:10.1111/j.1467-789X.2012.01046.x 


\section{Figures legends}

Figure 1. Experimental schedule. During the first week all animals received standard chow and water. After this habituation phase mice were randomly assigned to the group receiving standard chow (control), high-fat chow or a choice between a chocolate-mixture and standard chow. After 8 weeks (development phase) of continuous recording of feeding behavior, we limited the daily access to high-fat or chocolate-mixture to identify compulsive-like behaviors. SC $=$ Standard Chow, HF = High-Fat, CM = Chocolate-Mixture, Ltd = Limited

Figure 2. Exposure to high-fat or chocolate-mixture modifies feeding behavior. The figure shows feeding behavior parameters 8 weeks after the access to the high-fat (HF, left panel) or chocolate-mixture (CM, right panel) diet at the end of the development phase, (A) Body weight increase (\% from baseline) was more marked in mice exposed to high-fat food (left panel) than in those with free access to chocolate-mixture (right panel). (B) Energy intake in 24 hours $(\mathrm{kJ} / \mathrm{kg}$ ) was also higher in the HF mice group (left) than in the CM group (right), in which energy was mainly provided by chocolate-mixture meals (inset, right panel). (C) In the HF group (left) this increased energy intake is due to an increase in food intake (g/kg) that was not detected in the CM group, in which food intake did not differ from control mice. The inset also shows no significant differences between intake of standard chow and of chocolate-mixture in the CM group. (D) HF and CM mice showed increased number of meals along with a reduced (E) average meal duration (min), especially for chocolate meals in the CM group (E, right panel inset). (F) Eating rate (mg/s) was increased in both high-fat and chocolate-mixture animals, being again chocolate meals more affected than standard chow meals in the CM group (F, right panel inset), while (G) satiety (minutes until next meal per kJ consumed) was reduced only in 
HF mice. Data are expressed as mean \pm SEM. $\star \star \star P<0.001, \star \star P<0.01$, one-way ANOVA. SC = Standard Chow, $\mathrm{HF}=$ High-Fat, $\mathrm{CM}=$ Chocolate-Mixture.

Figure 3. Phenotypic array of feeding behaviors in mice exposed to high-fat diet. The arrays represent the fold changes of different feeding/drinking behaviors in the habituation phase (A) and over the development phase of obesity (B) showing each square the values of each week. Red corresponds to behaviors that increase their frequency (fold change $>1$ ), and green to behaviors that decrease their frequency (fold change $<-1$ ) in high-fat animals. Color intensities represent the amount of phenotypic change with no changes depicted in black. Behaviors include average intake in each drinking/eating event, number of drinking/eating events, average duration, drinking/eating rate and average time between drinking/eating events. This phenotypic representation provides an easy way of detecting the behavioral changes along time. Note the rapid increase in intake, meal number and eating rate, along with the strong and immediate decrease in meal and intermeal interval duration upon exposure to high-fat. (A) Habituation phase. (B) Feeding parameters of HF mice compared to control animals during the obesity development phase. $\star \star \star P<0.001, \star \star P<0.01, \star P<0.05$, Wilcoxon rank-sum test.

Figure 4. Phenotypic array of feeding behaviors in mice with free access to a chocolatemixture. Fold changes of feeding/drinking behaviors are represented in the habituation phase (A) and over the development of obesity (B). Red corresponds to behaviors that increase their frequency (fold change $>1$ ), and green to behaviors that decrease their frequency (fold change $<-1$ ) in CM animals. Each square corresponds to one week. Color intensities represent the amount of phenotypic change with no changes depicted in black. Behaviors include average 
intake in each drinking/eating event, number of drinking/eating events, average duration, drinking/eating rate and average time between drinking/eating events. (A) Habituation phase. (B) Standard chow and (C) Chocolate-mixture consumption parameters of CM animals compared to control animals during the obesity development phase. $\star \star \star P<0.001, \star \star P$ $<0.01, \star P<0.05$, Wilcoxon rank-sum test. $\mathrm{SC}=$ Standard Chow, $\mathrm{CM}=$ Chocolate-Mixture.

Figure 5. Longitudinal behavioral recordings show early disruption of circadian meal pattern and indicate binge-like eating behavior in high-fat exposed mice. The figure depicts the feeding bouts along time of 9 control (gray) and 8 mice receiving high-fat chow (black). Each line represents feeding bouts of individual mice as rectangles, the height of the bar indicating the amount consumed during 30 minutes in grams (g). Intakes corresponding to control mice are depicted in the upper part of the graph and those corresponding to HF mice in the bottom part. Gray shadow areas are dark (active) periods, also represented as blue lines (light-dark phase track). Metadata also continuous periods of recording corresponding to a single recording track depicted as red or yellow segments (contained in a file). (A) During the habituation phase, in which all animals are given standard chow, the meal pattern was equal in all groups. (B) The arrow marks the introduction of the high-fat diet and the early stages of the obesity development phase. Note the loss of circadian eating rythmicity very early upon introduction of the high-fat chow. (C) HF animals showed an intake increase (marked with red boxes) after cage cleaning in which animals have no access to food ('cleaning deprivation'). Remarkably, this pattern is not detected in controls. Ctrl = Control, HF = High-Fat, LD = Light-Dark.

Figure 6. Longitudinal representation of behavioral recordings depicts an early disruption in CM mice. Tracks represent mouse individual feeding behaviors. Food intakes during 30 minutes are shown as rectangles: height of the bar indicates the amount consumed during the 
30 minutes period. Intakes corresponding to controls mice are depicted in gray and those corresponding to CM mice in black (A and B panels). On C panel, CM mice intake is separated into intakes corresponding to standard chow (gray) and chocolate-mixture (black). On the bottom there are two different tracks: file track consists in segments colored as red or yellow corresponding to a single recording (contained in a file). At the same time phase track is colored to show dark (dark blue) and light (light blue) phases of the circadian cycle. For the sake of simplicity mice tracks parts corresponding to the dark phase are shadowed. (A) During the habituation phase in which all animals are given standard chow, the meal pattern was equal in the two groups. (B) Upon introduction of the chocolate-mixture diet, light phases tend to be more populated in comparison with their control littermates. (C) Although not significant, CM animals tend to eat chocolate during resting phases just after the introduction of the chocolatemixture food. Ctrl = Control, CM = Chocolate-Mixture, LD = Light-Dark.

Figure 7. HF mice showed binge eating-like behavior and food-taking inflexibility. Limited access to high-fat food induced a marked increase of high-fat food consumption after refeeding. HF mice showed (A) increased energy intake (kJ/kg), (A inset) caloric intake when only standard chow is available compared with basal high-fat consumption $(\mathrm{kJ} / \mathrm{kg})$, (B) eating rate (mg/s) and (C) meal size (g). CM mice also showed binge eating-like behavior and foodtaking inflexibility. The limited access to this diet induced a marked increase of chocolatemixture diet consumption during the 1 hour limited access. CM mice showed (D) increased energy intake $(\mathrm{kJ} / \mathrm{kg})$ and $(\mathbf{E})$ meal size (g) whereas eating rate was unchanged (mg/s) (F). Interestingly, (D inset) chocolate-mixture animals did not significantly increase standard chow energy intake to compensate the energy unbalance during the 23 remaining hours of the test during which mice have only access to standard chow. Data are expressed as mean \pm SEM of 
each parameter in one hour. $\star P<0.05$, one-way ANOVA. Ltd $=$ Limited, HF $=$ High-Fat, $\mathrm{CM}=$ Chocolate-Mixture . 

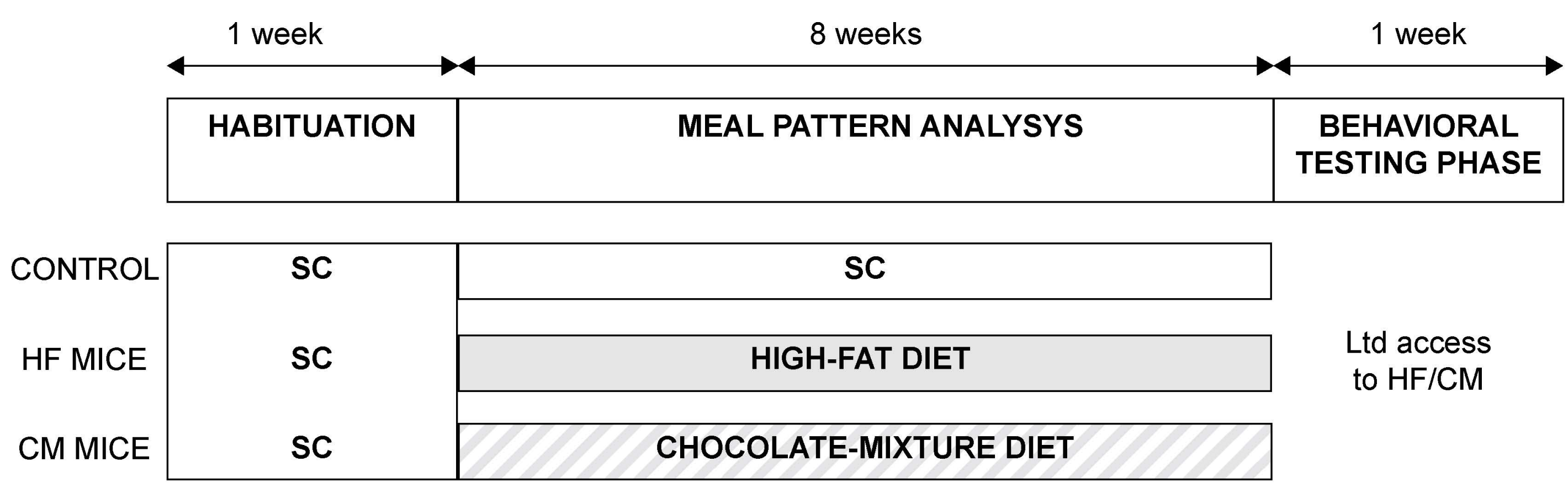

Ltd access to HF/CM 
A

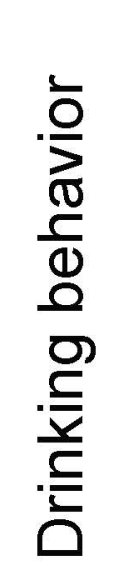

흘

Average intake

Number of drinks

Average duration

Drinking rate

Average interdrink duration - - - - - - - - - - Average intake

Number of meals

Average duration

Eating rate

Average intermeal duration
B

HF mice
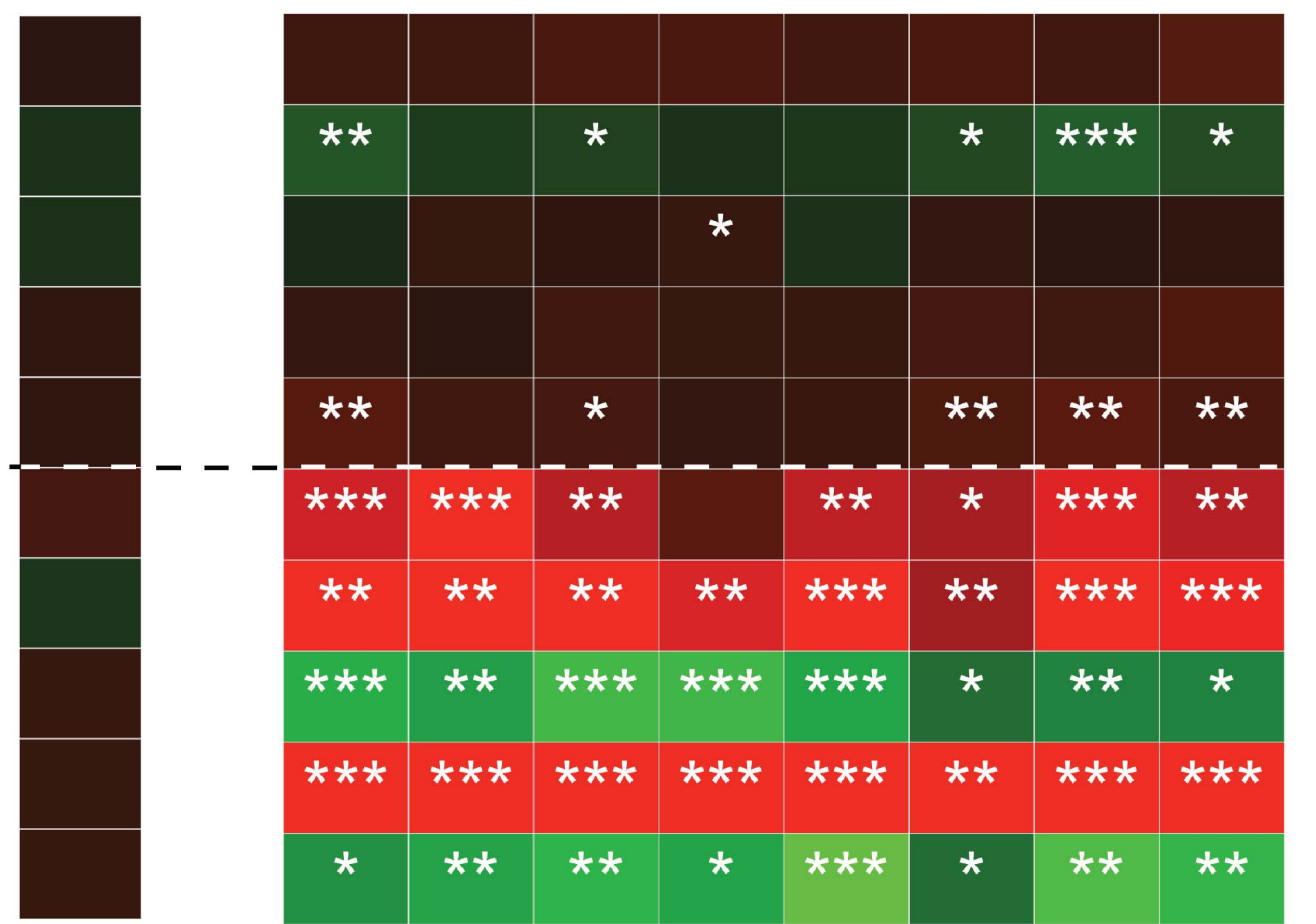
$--$

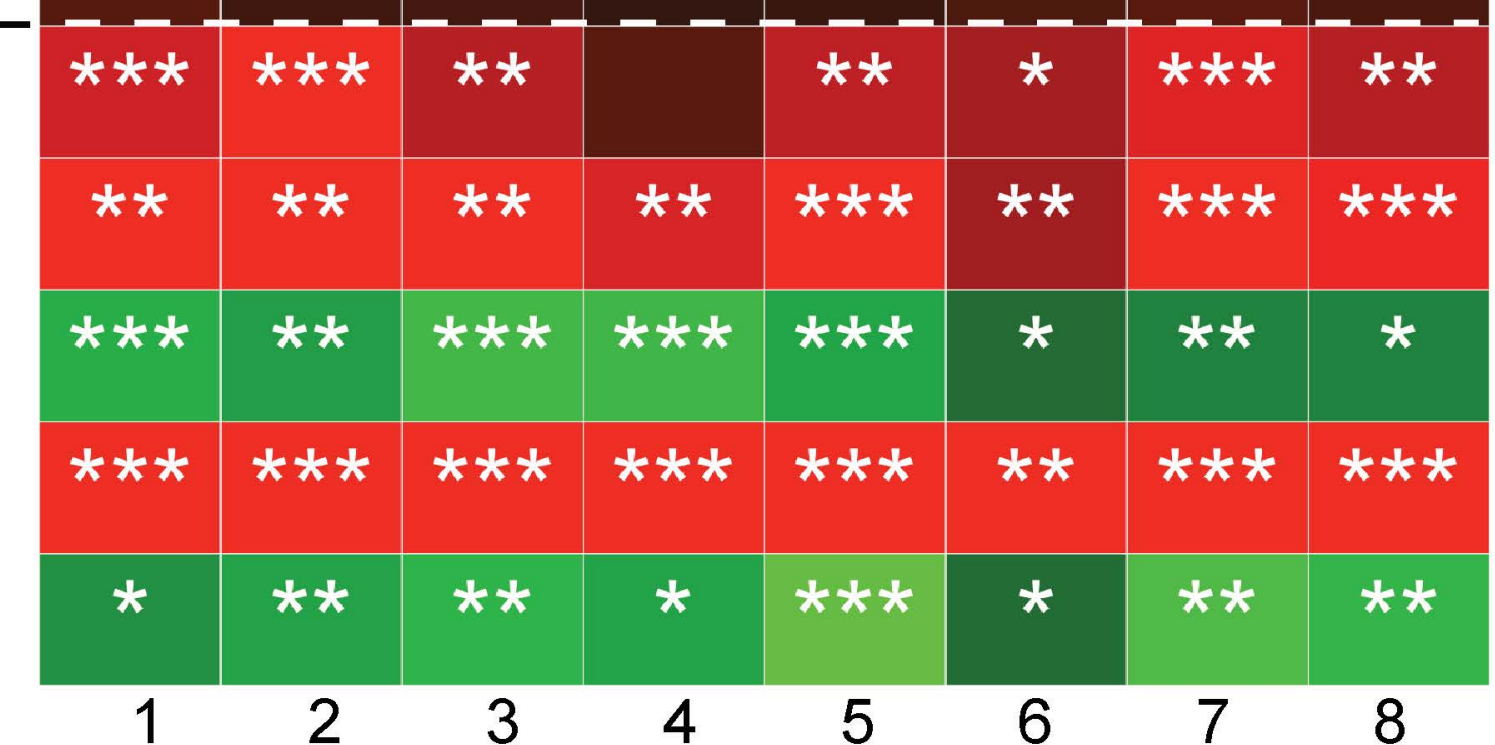

Habituation phase

Obesity development phase (weeks)
Fold change

$>3$

2

1 
SC feeder

C

$\mathrm{CM}$ feeder

Average intake

Number of drinks

Average duration

Drinking rate

Average interdrink duration - - - - - - - - - Averag intake

Number of meals

Average duration

Eating rate

Average intermeal duration

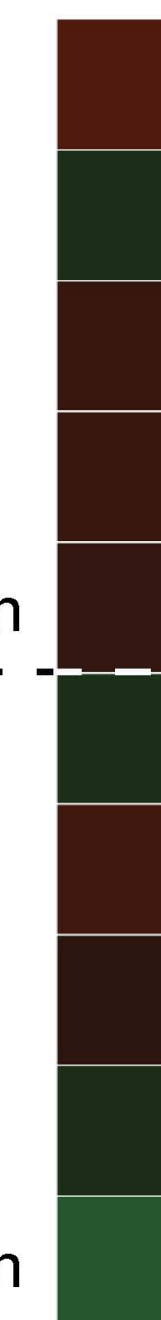

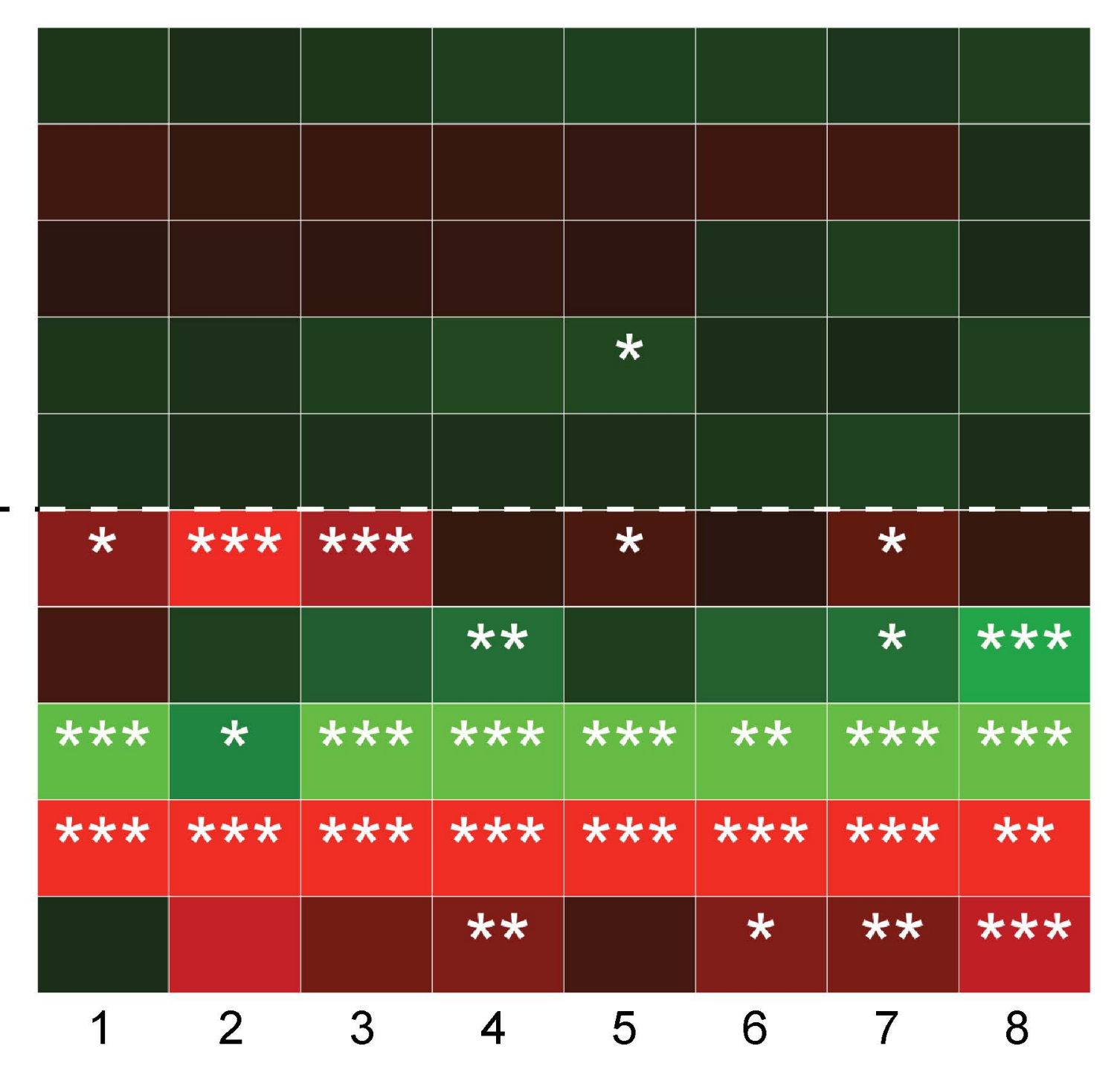

Fold change

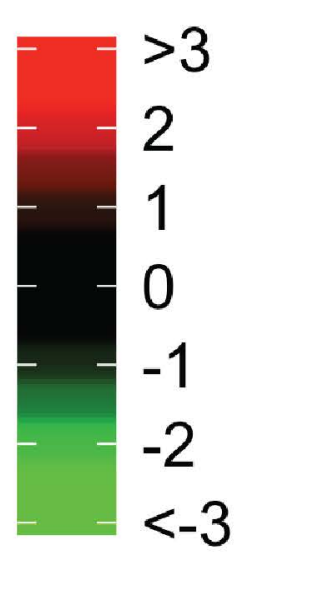


Ctrl 1

Ctrl 2

Ctrl 3

Ctrl 4

Ctrl 6

Ctrl 7

Ctrl 8

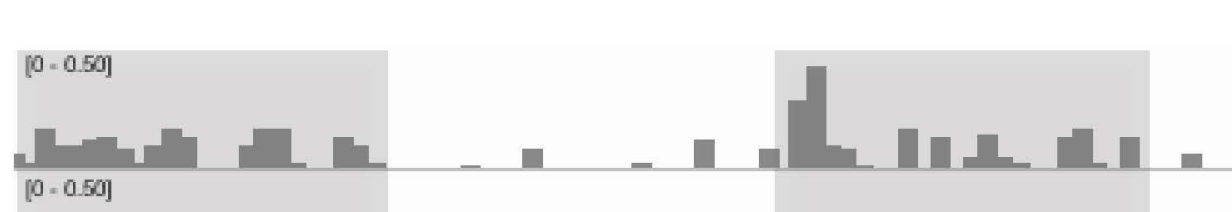
Nㅐㄷ. .I $\frac{-10.050]}{10.050}-1+1$ then 1. n oninal L.

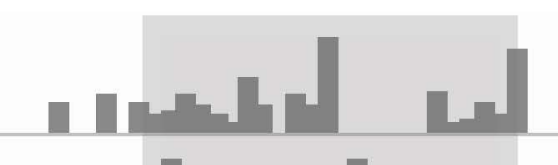
[10.080] ... II

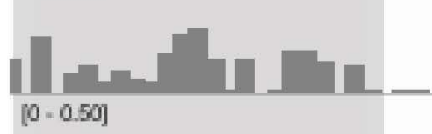

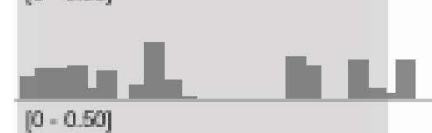

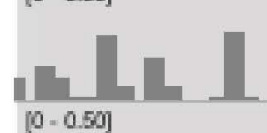
|

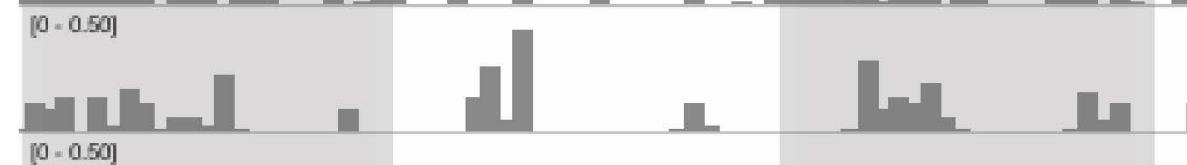

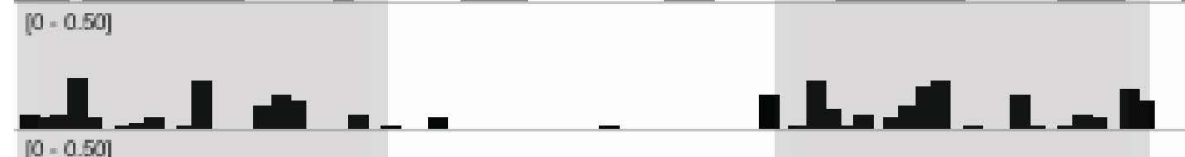

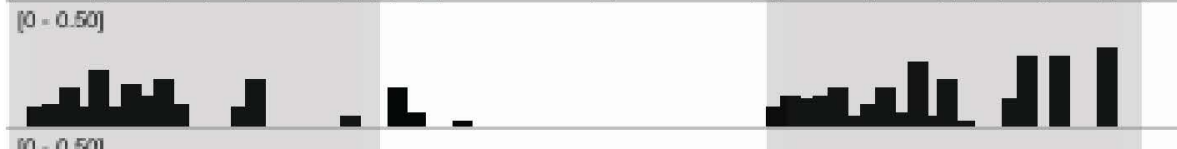

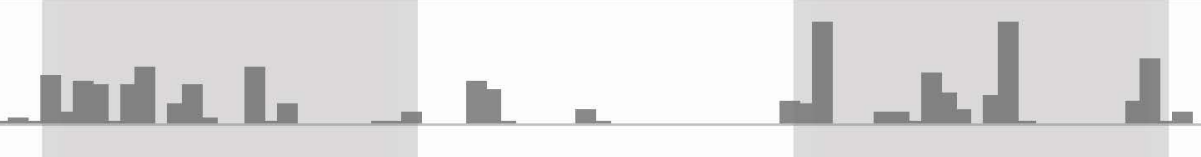

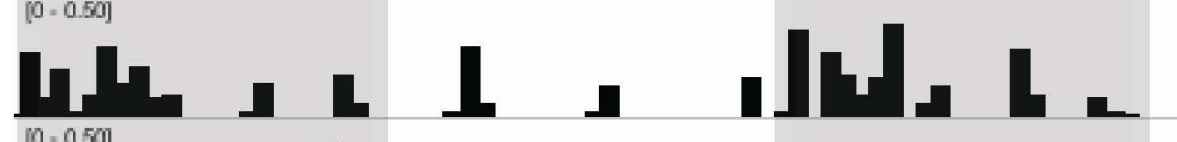

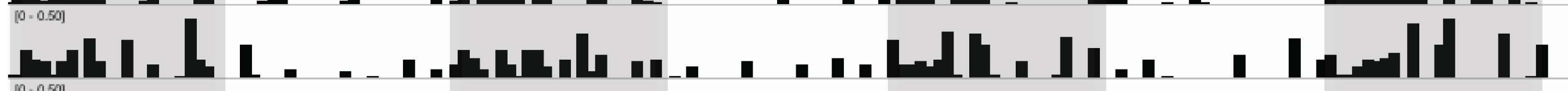

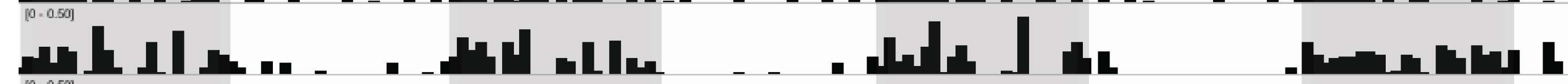

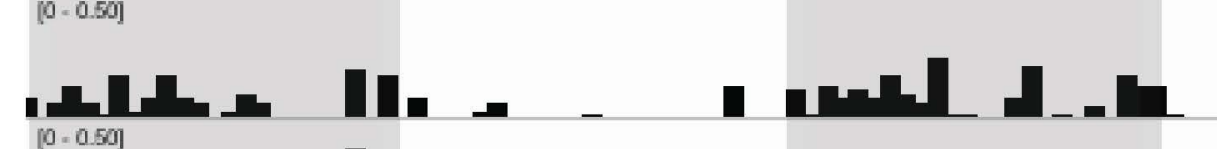

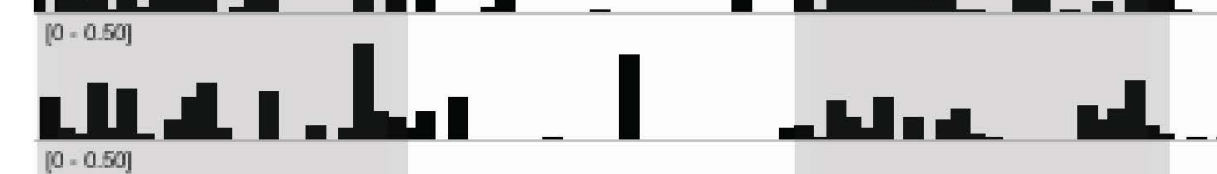

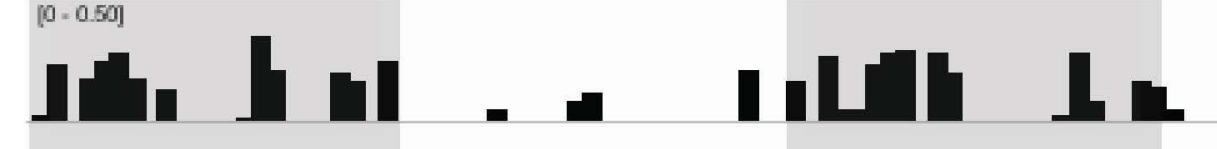
- 1 - 14

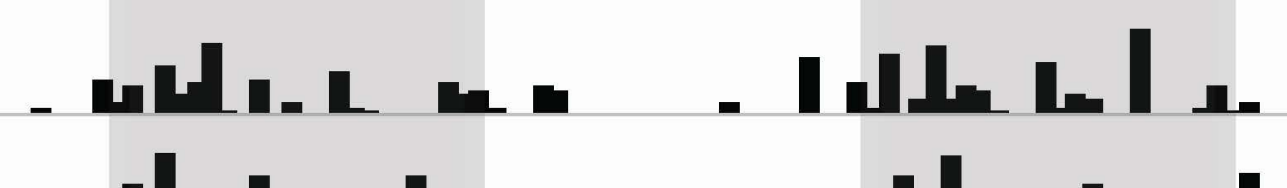

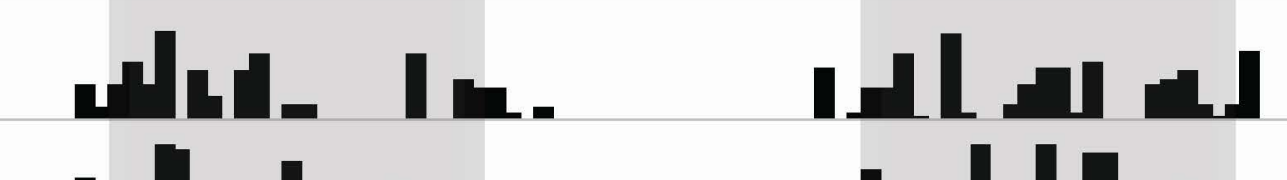

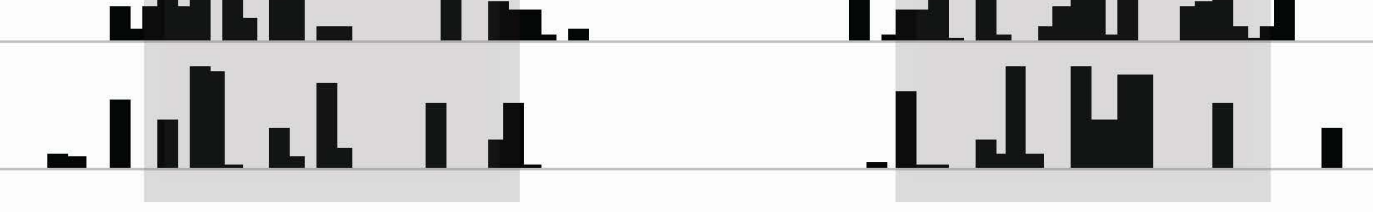

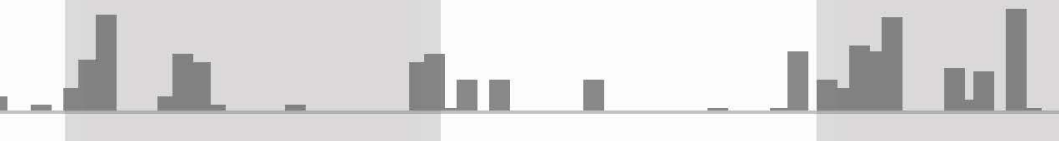

. 1 -

o난 I I

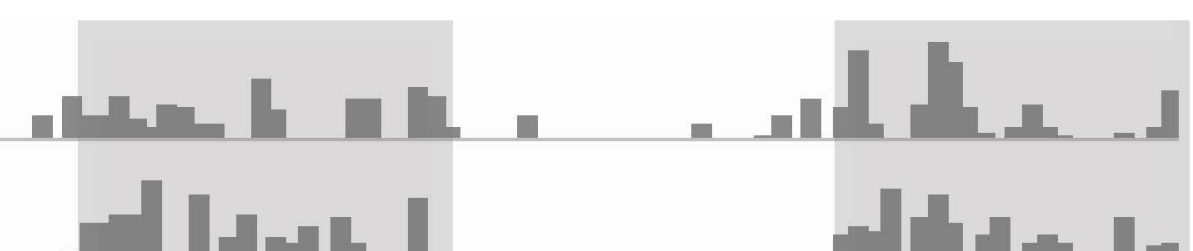

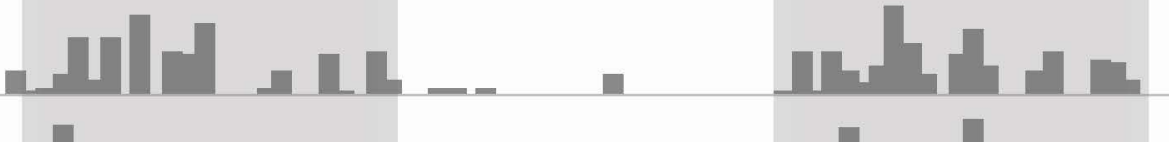

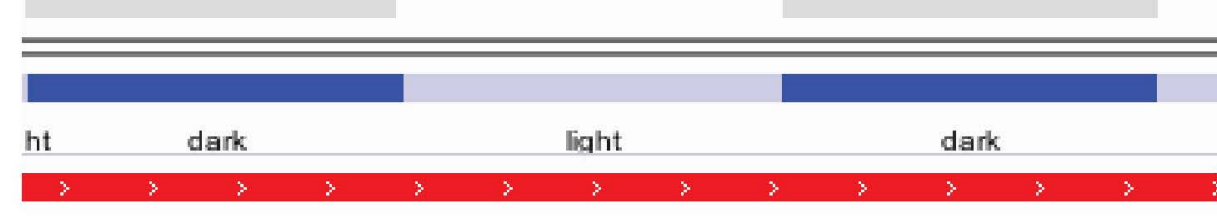

B

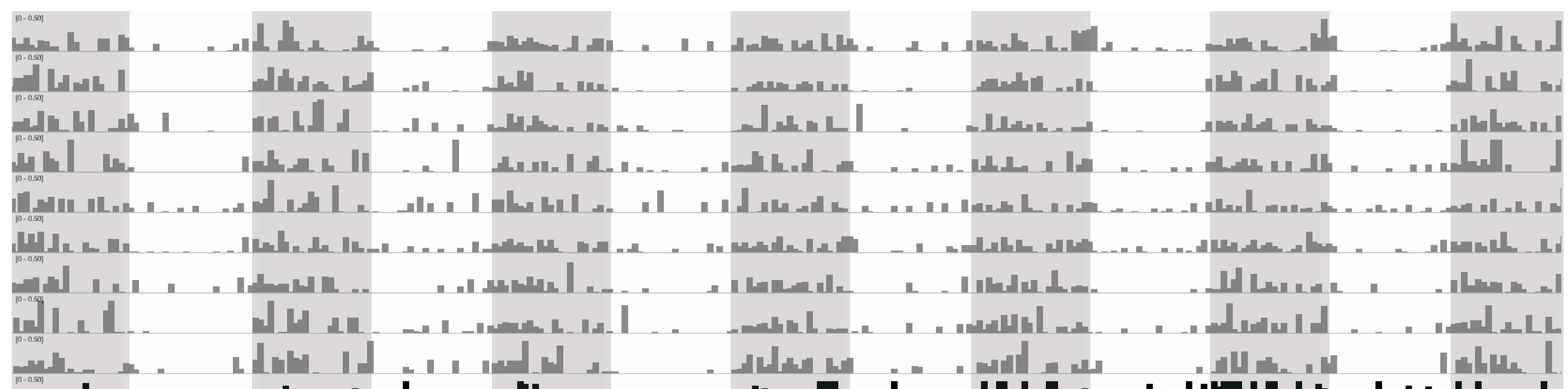

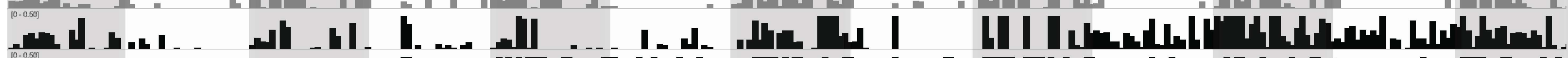

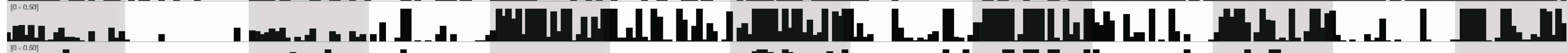

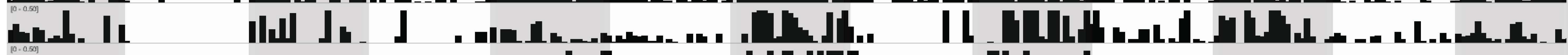

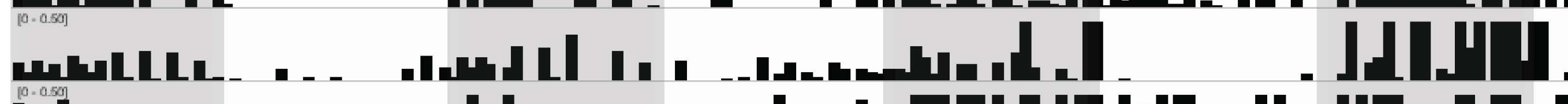

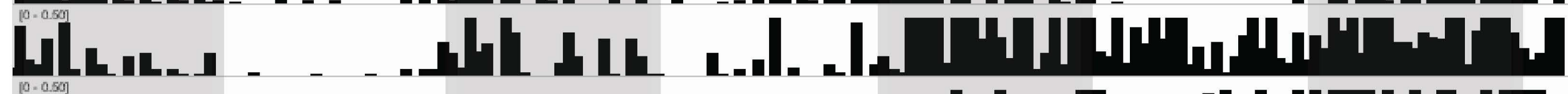

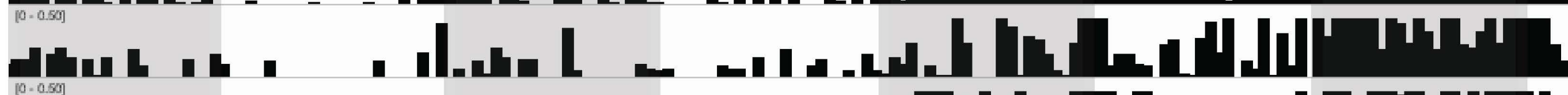

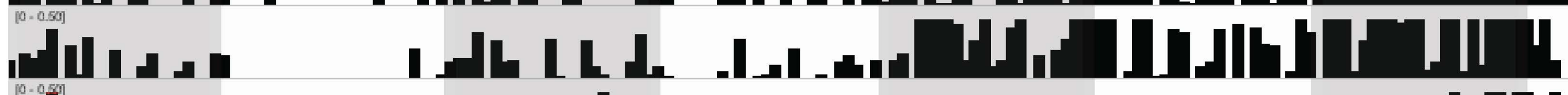

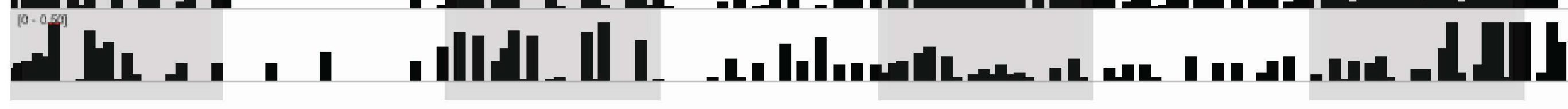

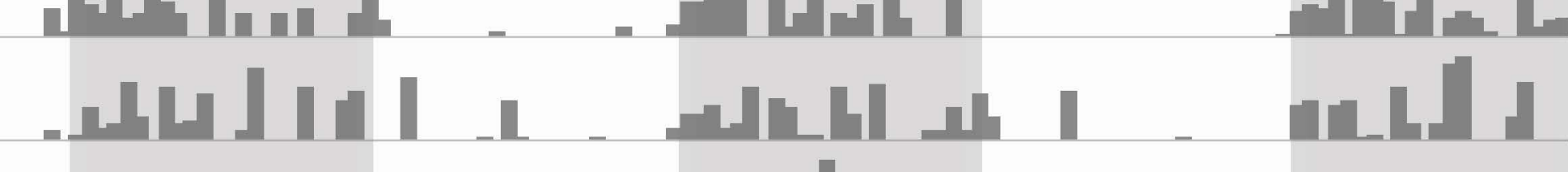

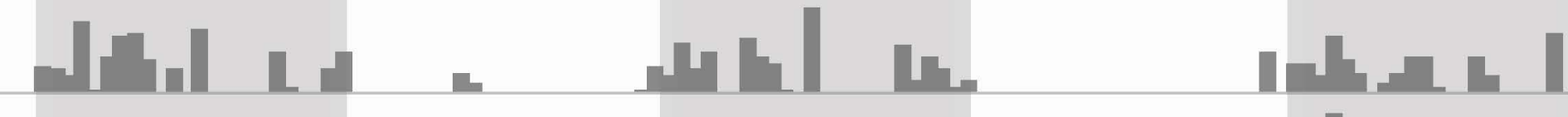

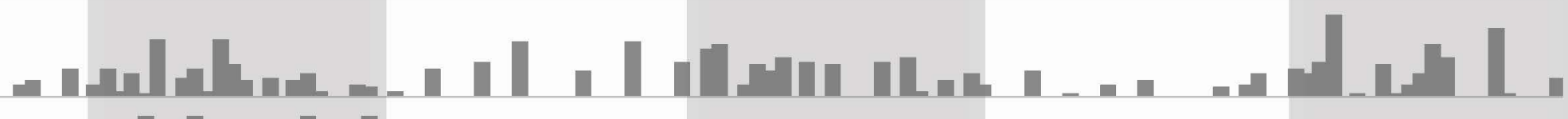

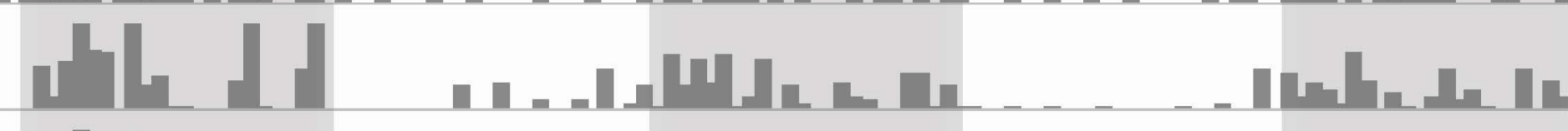

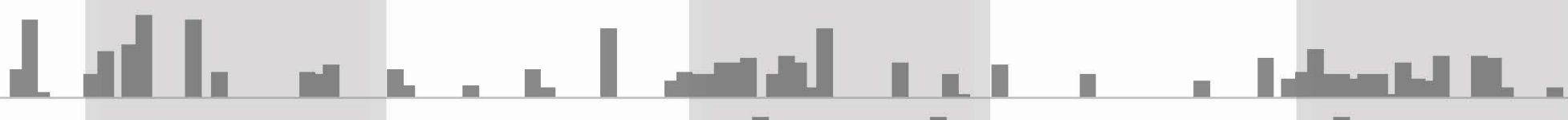

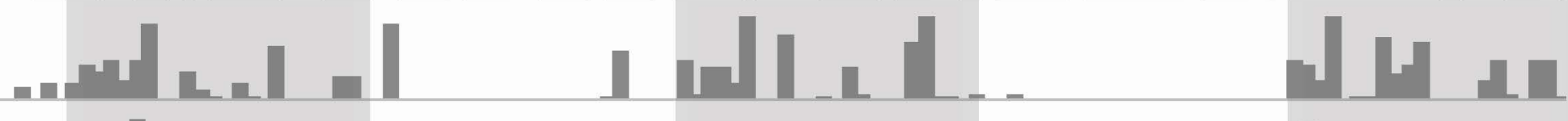

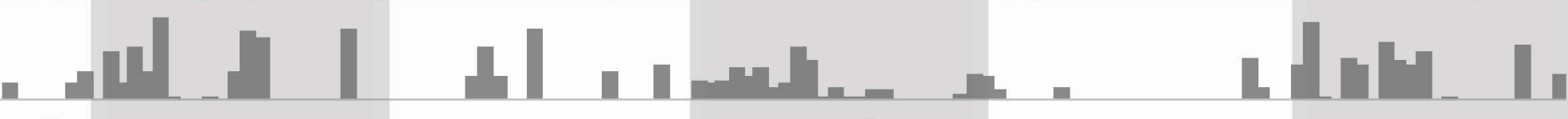

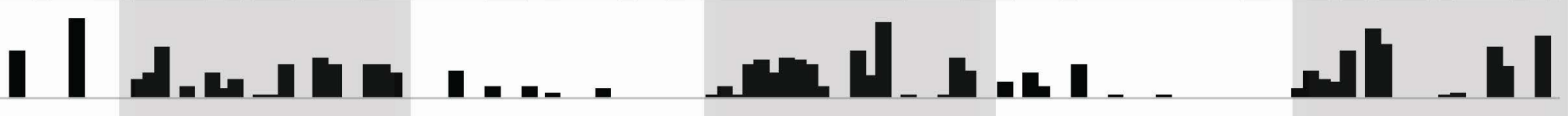

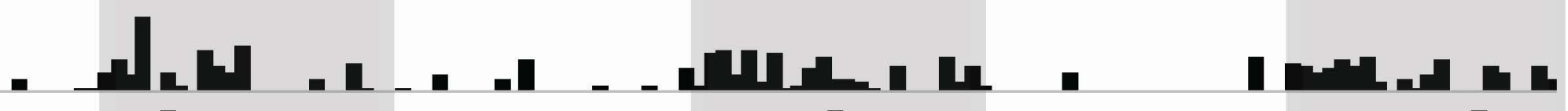

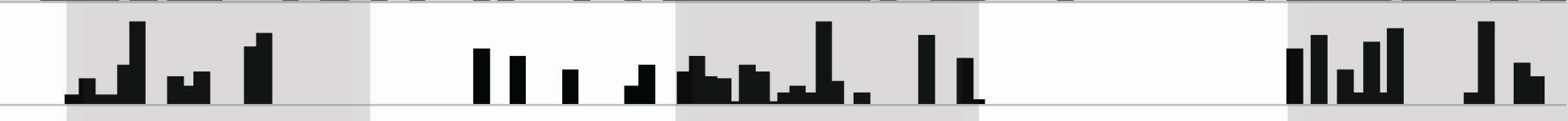

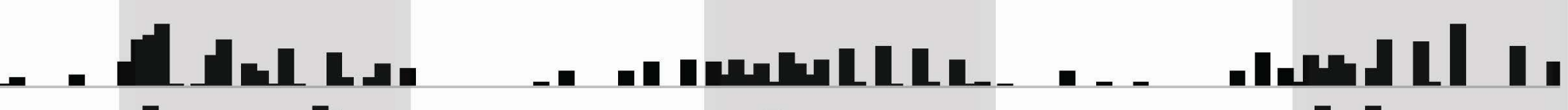

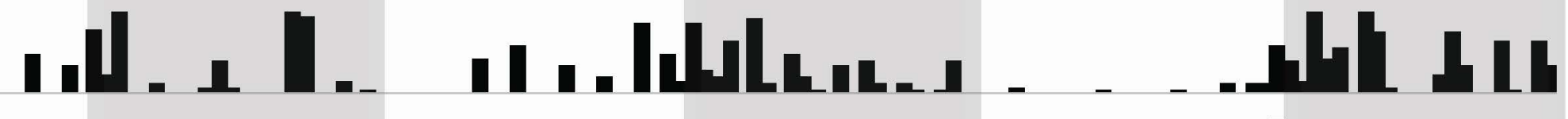

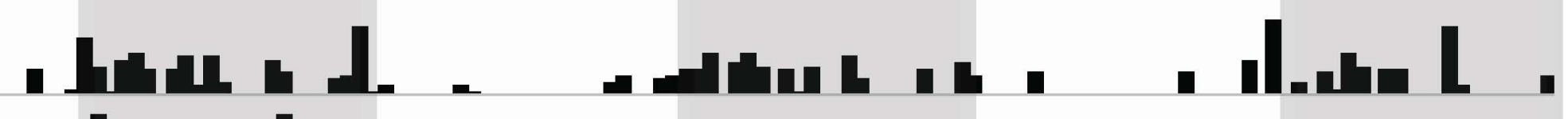

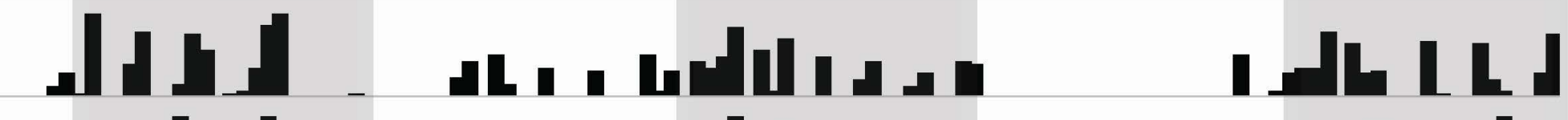

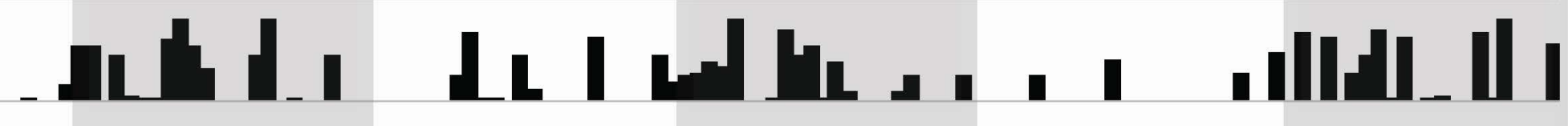

I H.VI hd |

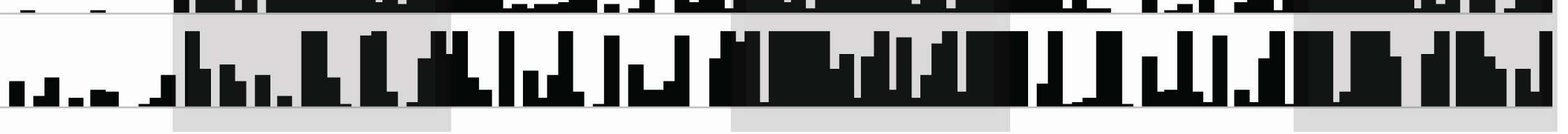

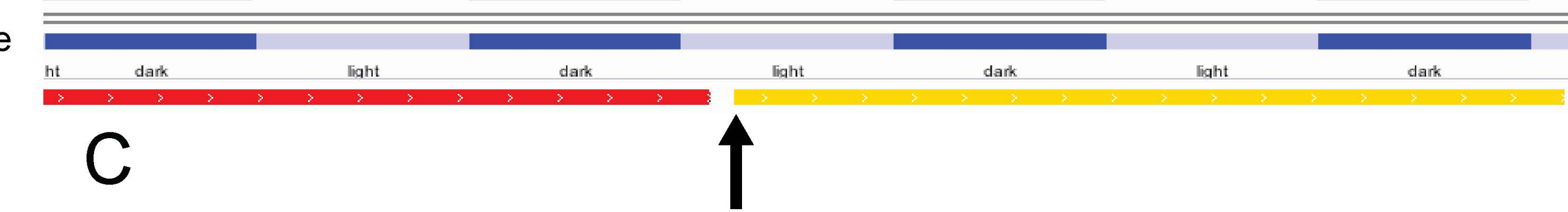
|

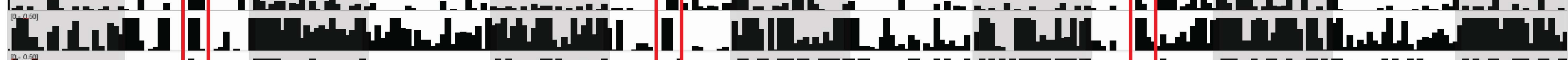

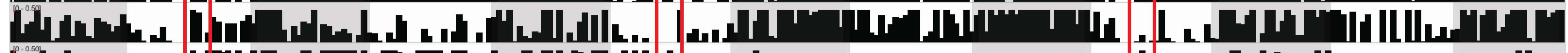

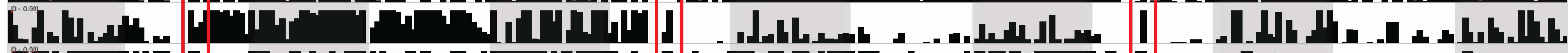

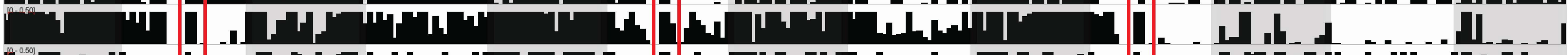

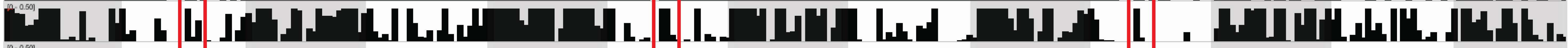

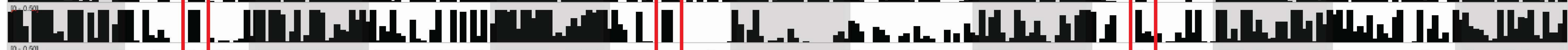

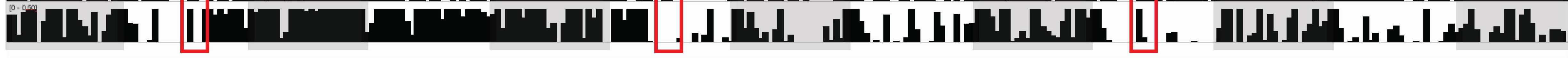




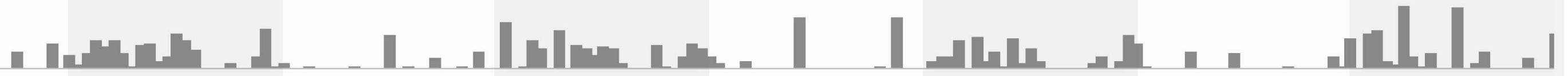

III l. Ph

.IIJ w d.

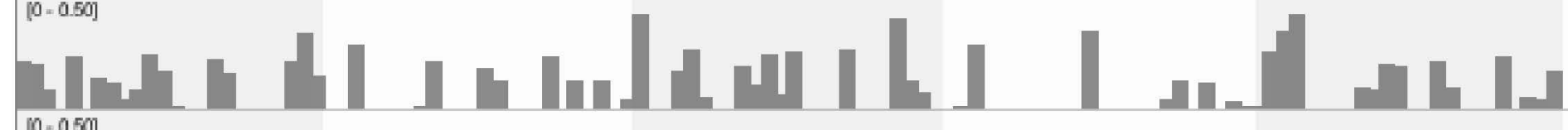

III L-IL | | |l| || |L

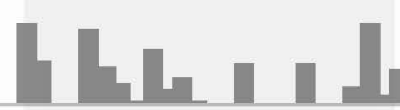

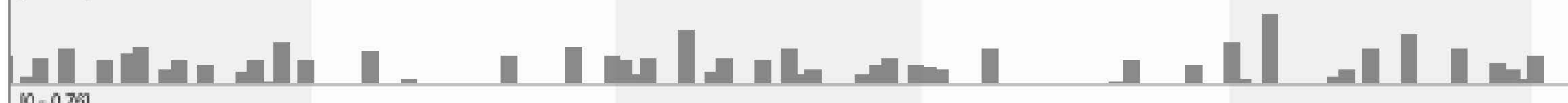

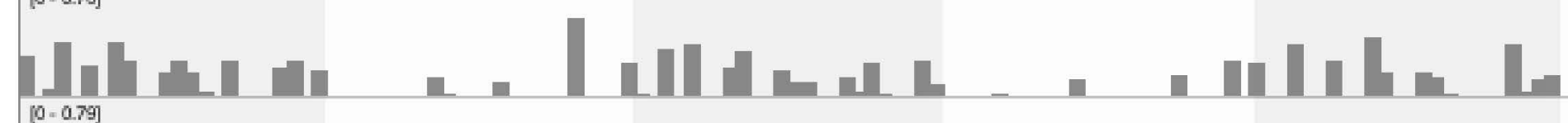

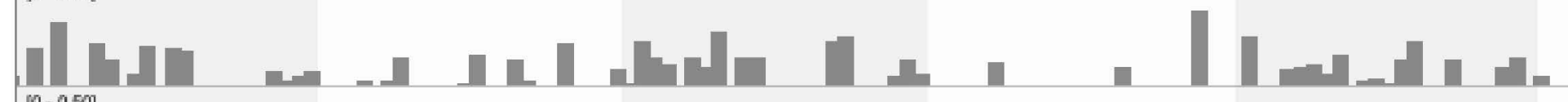

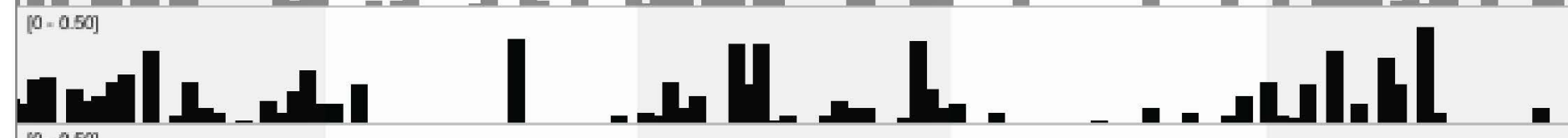

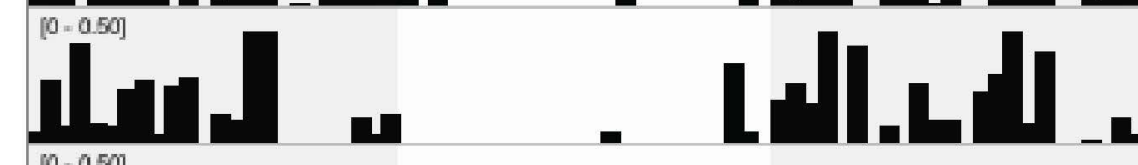

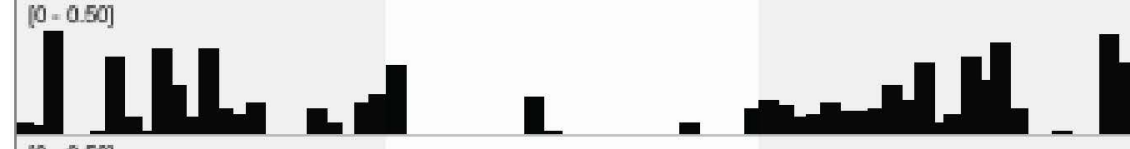

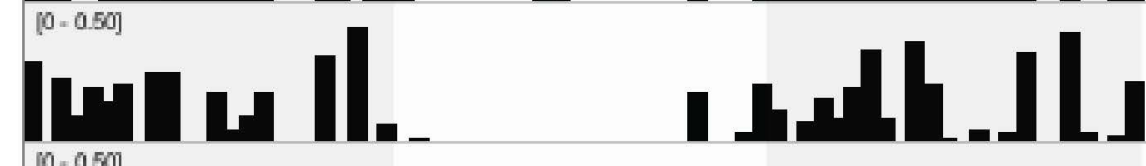

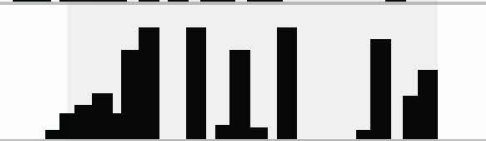

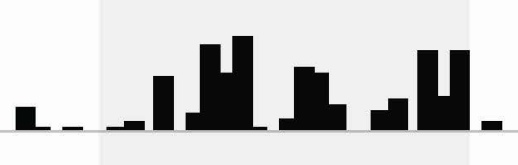

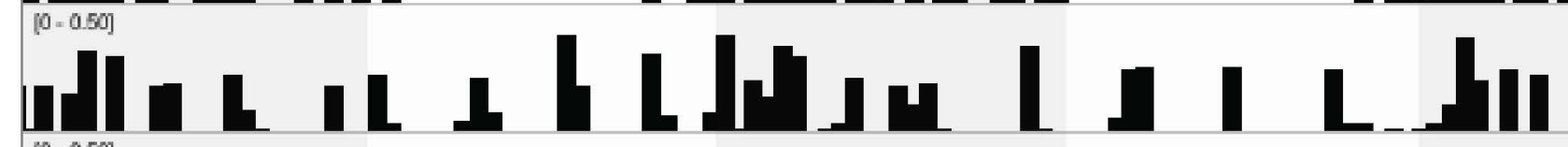

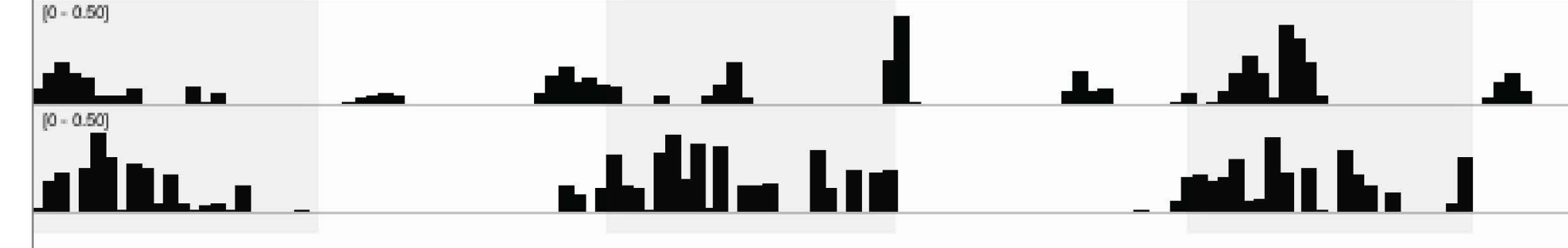

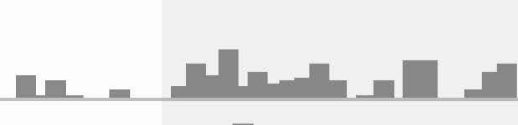

| Lال

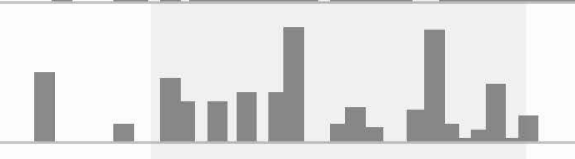
H. .

Ih lhenth

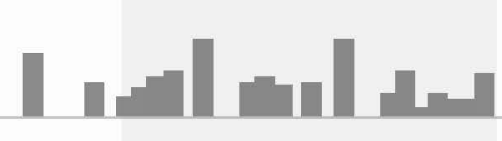

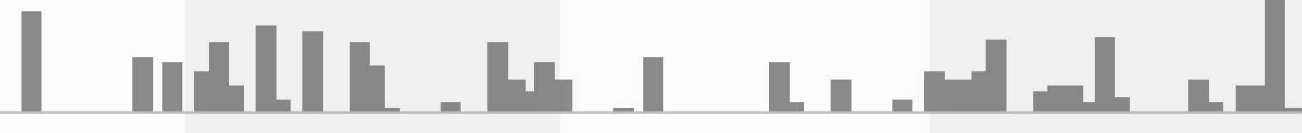

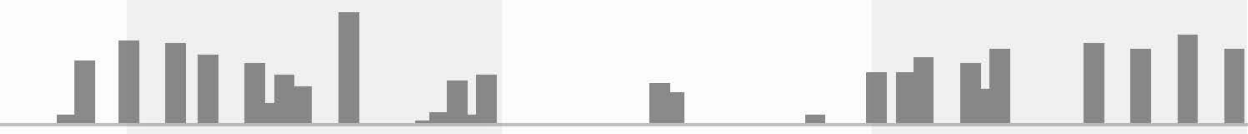

\section{|| | |}

I.

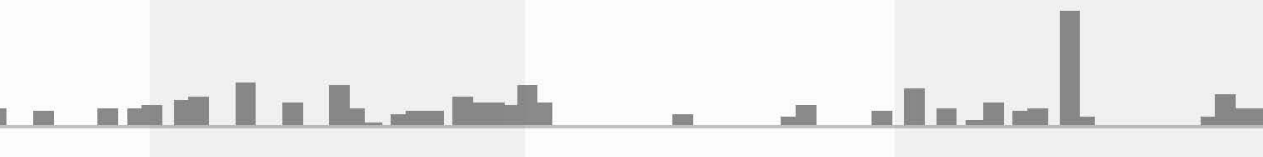

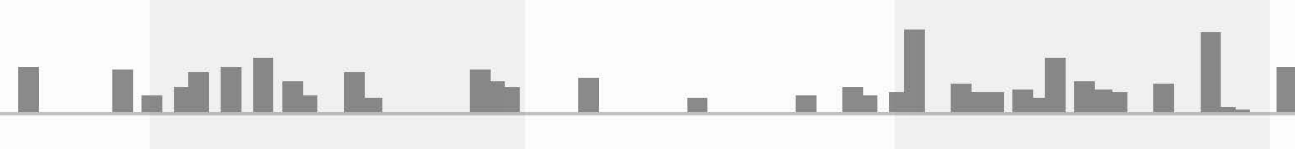

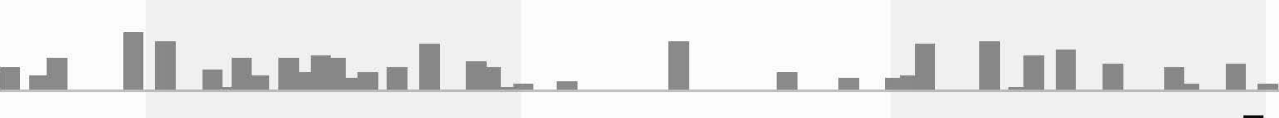

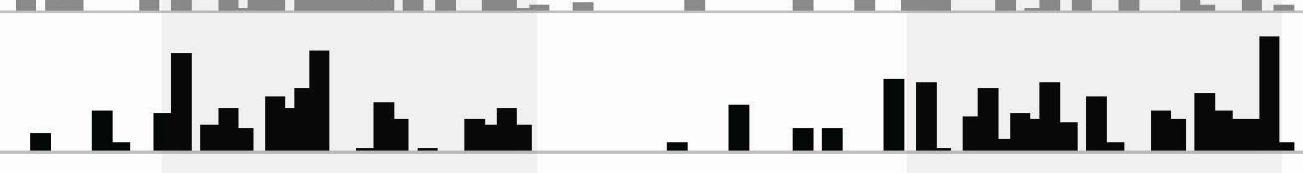

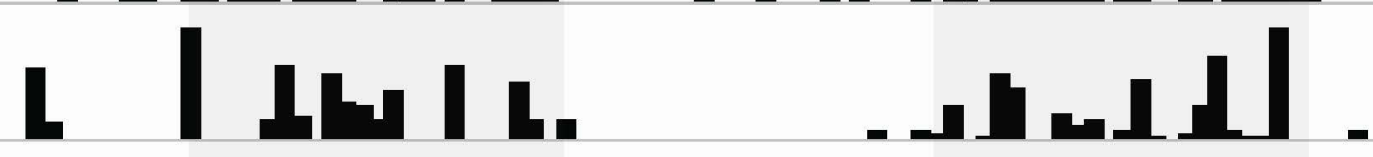

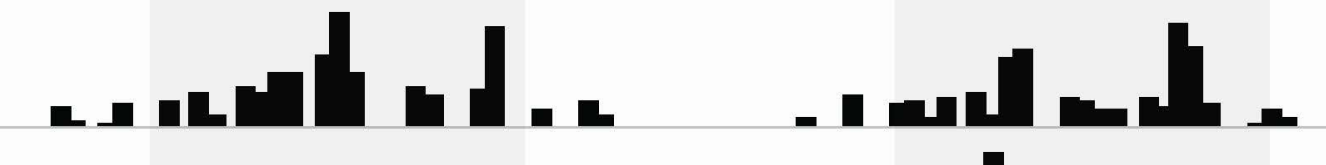

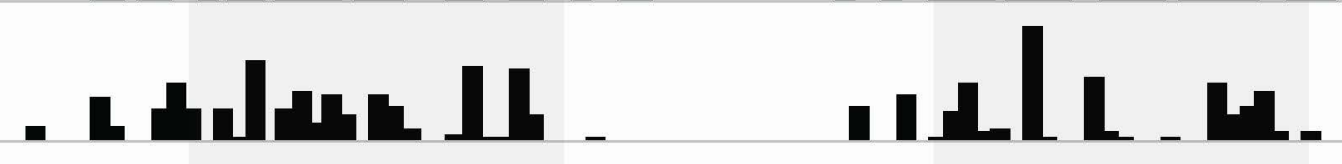

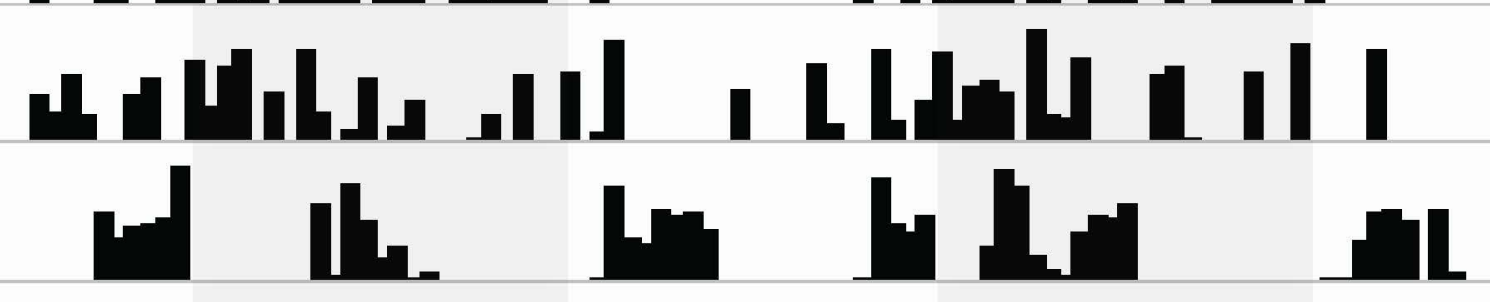

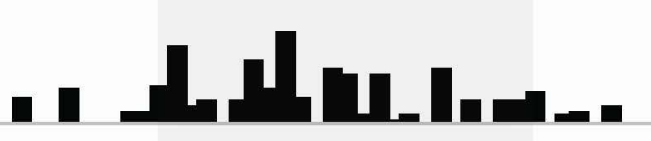

\section{h_mind I I}

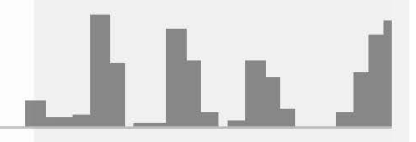

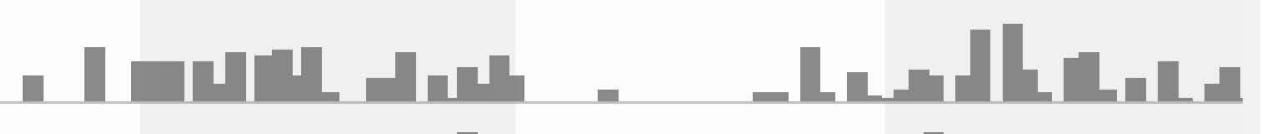

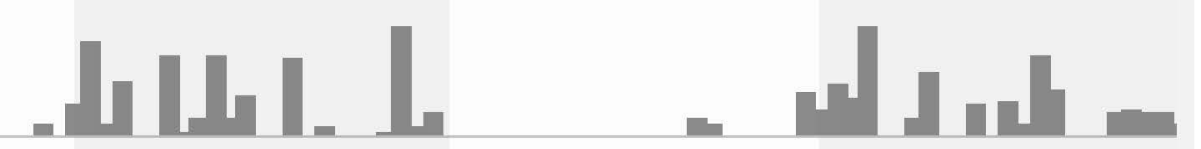

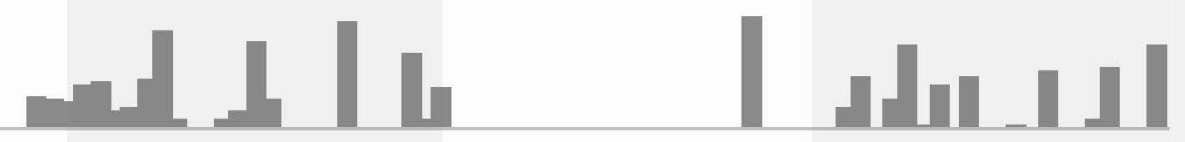

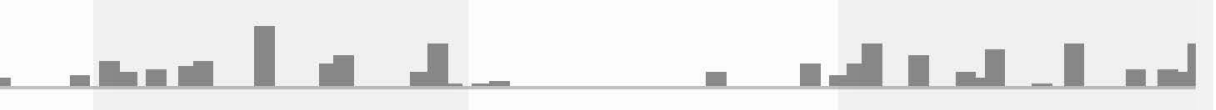

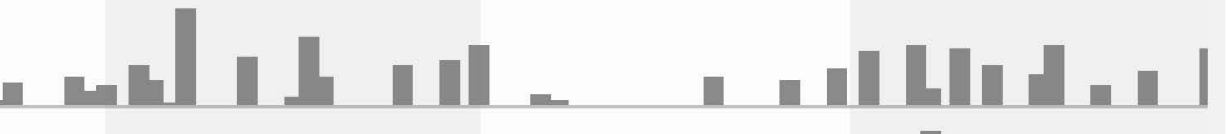

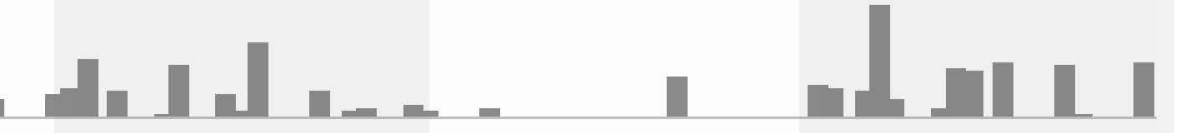

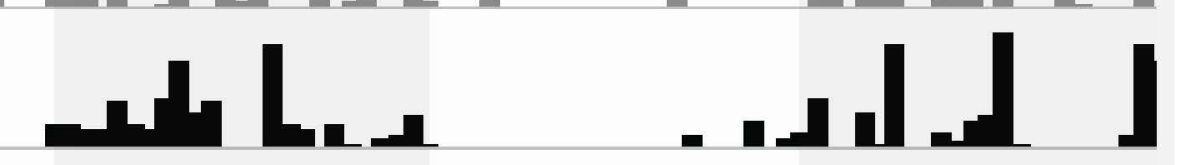

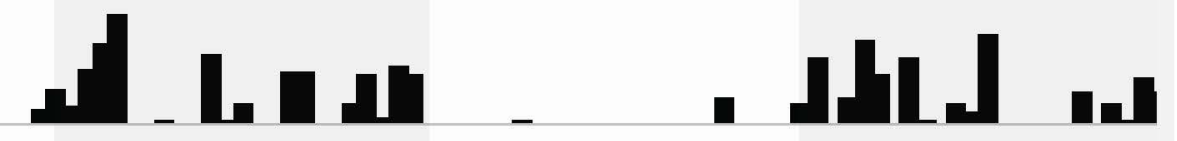

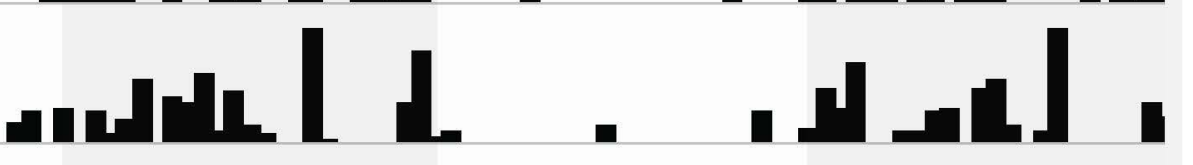

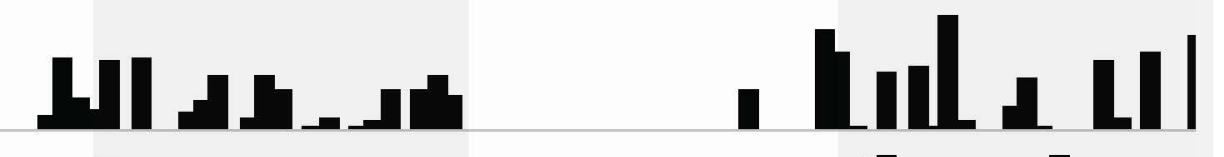

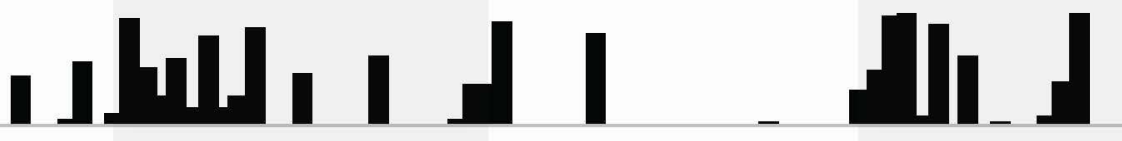

\section{ind}

I L L I I
Iter L

B

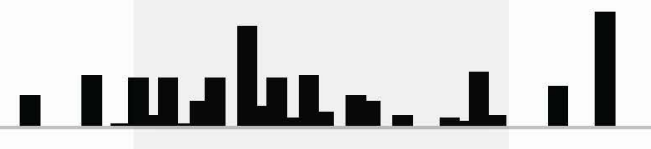

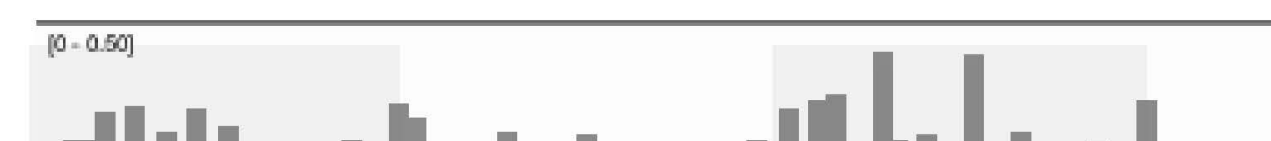

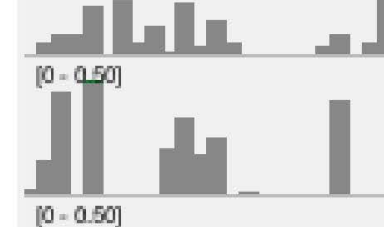

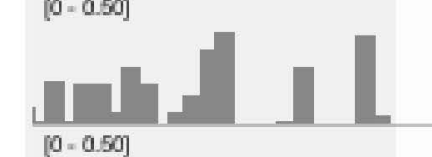

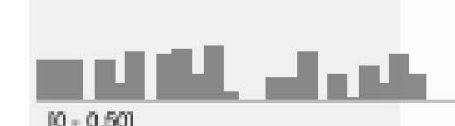

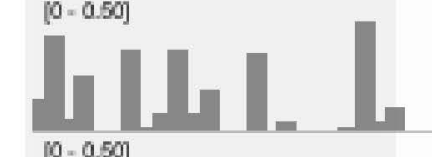
.

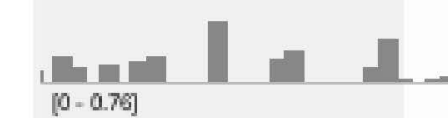

I. I I I I I III -

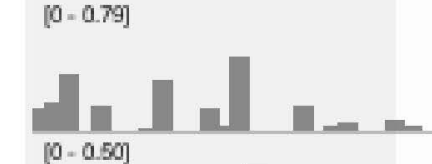

10.050)

1.

$\underset{[0.0 .050]}{[0.058]}$

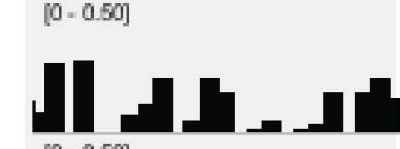

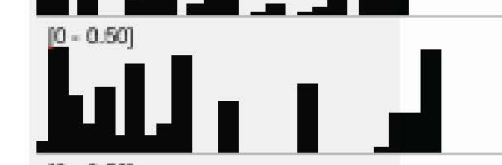

\section{[1]}

- Let. 1. In -

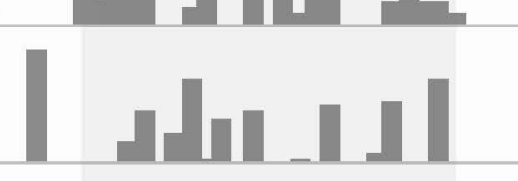

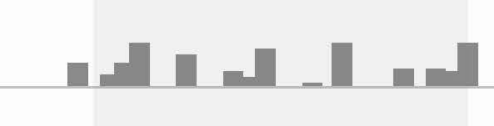

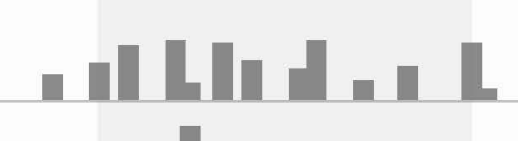

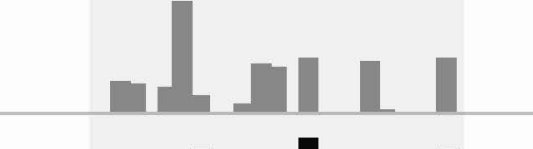

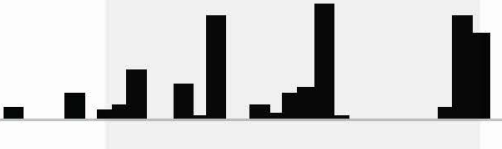

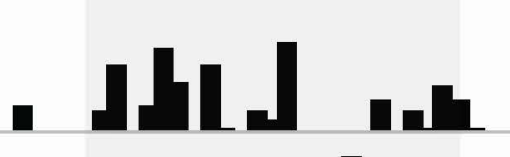

I. 1 thes

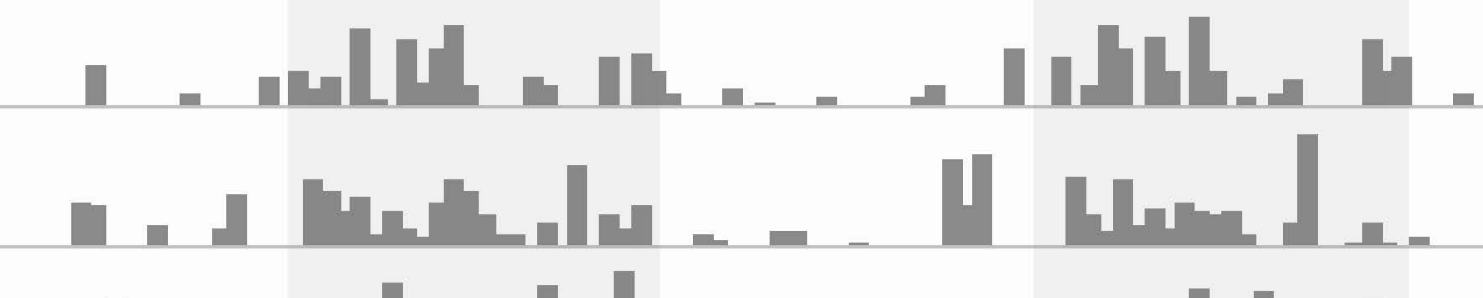

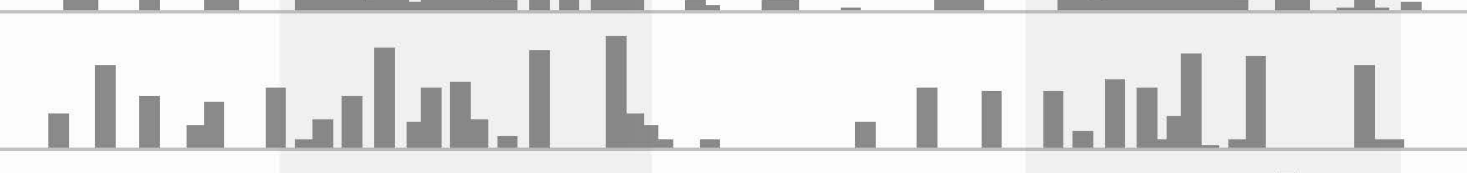
| millank

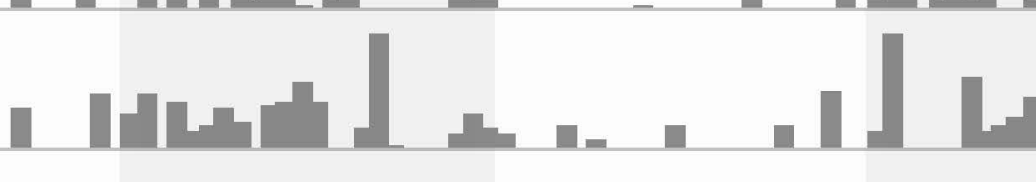
1. III

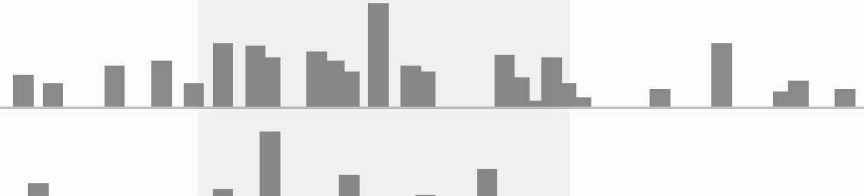

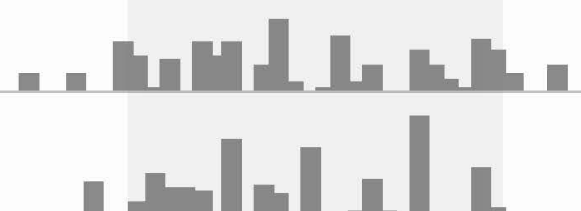
InIIL

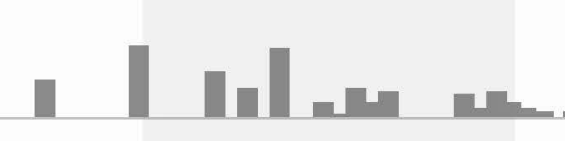

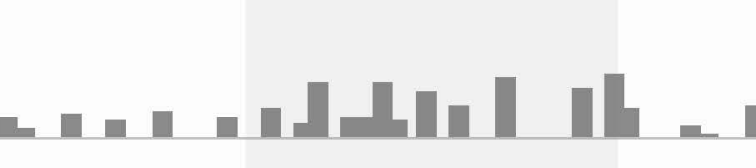
- and -

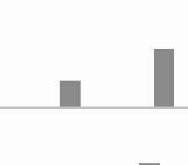

1
IILLL1.

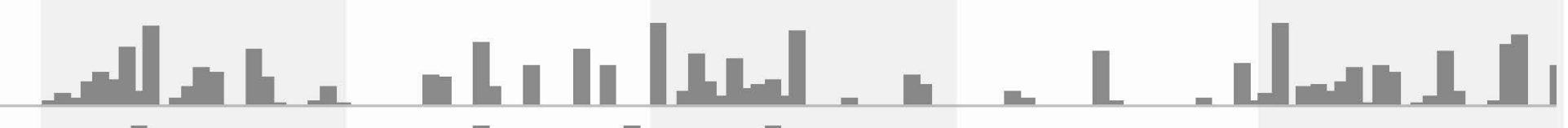

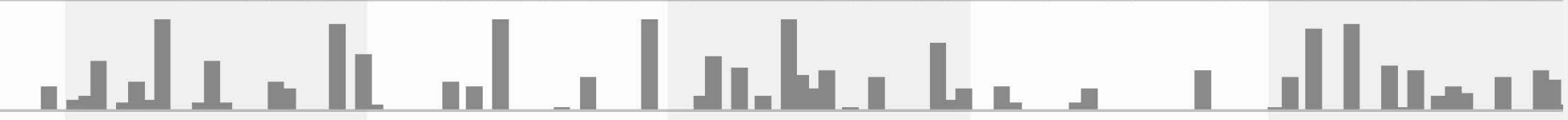

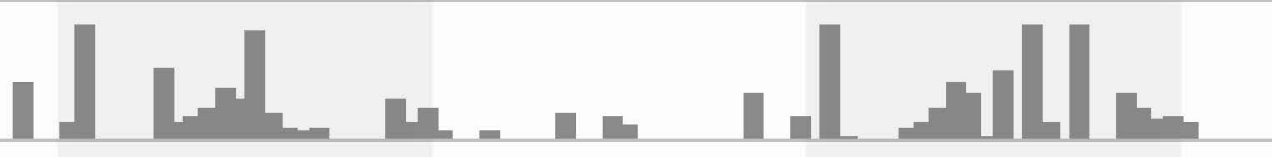

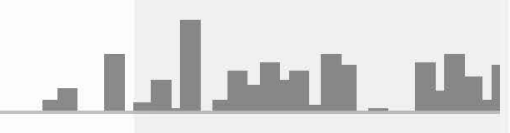

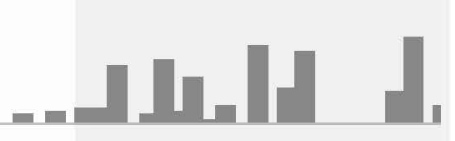
|hill d $\mathrm{C}$

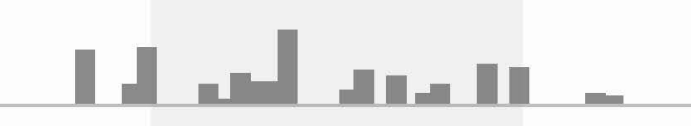

inal.

$1+1.1$

(1)

inting

1.

|ndrint

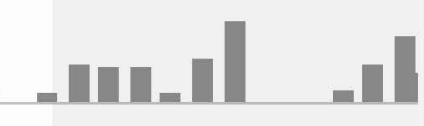

Control (Ctrl) mice Chocolate-mixture (CM) mice

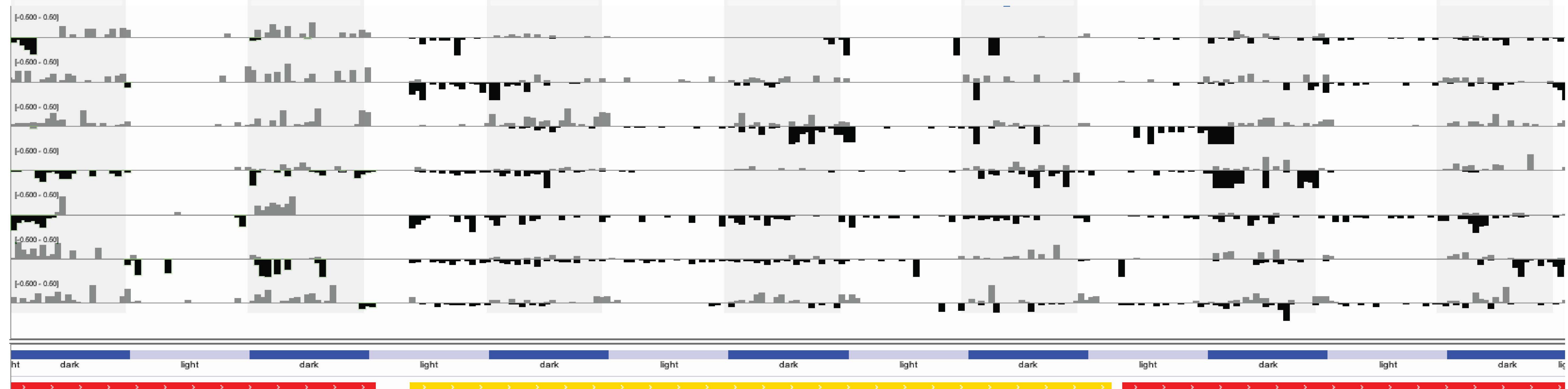


A Energy intake in $1 \mathrm{~h}$ Itd access to HF

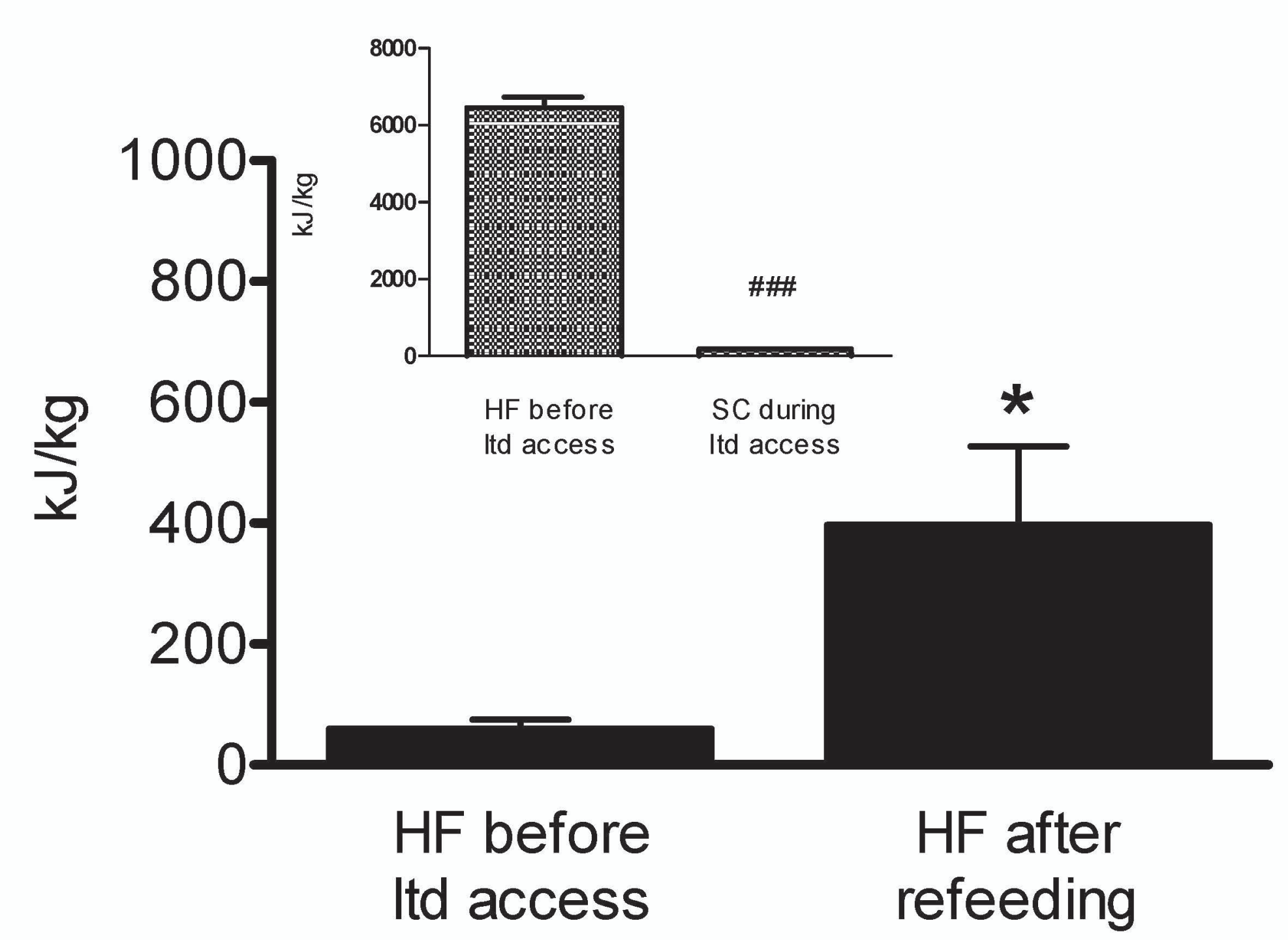

D Energy intake in $1 \mathrm{~h}$ Itd access to CM

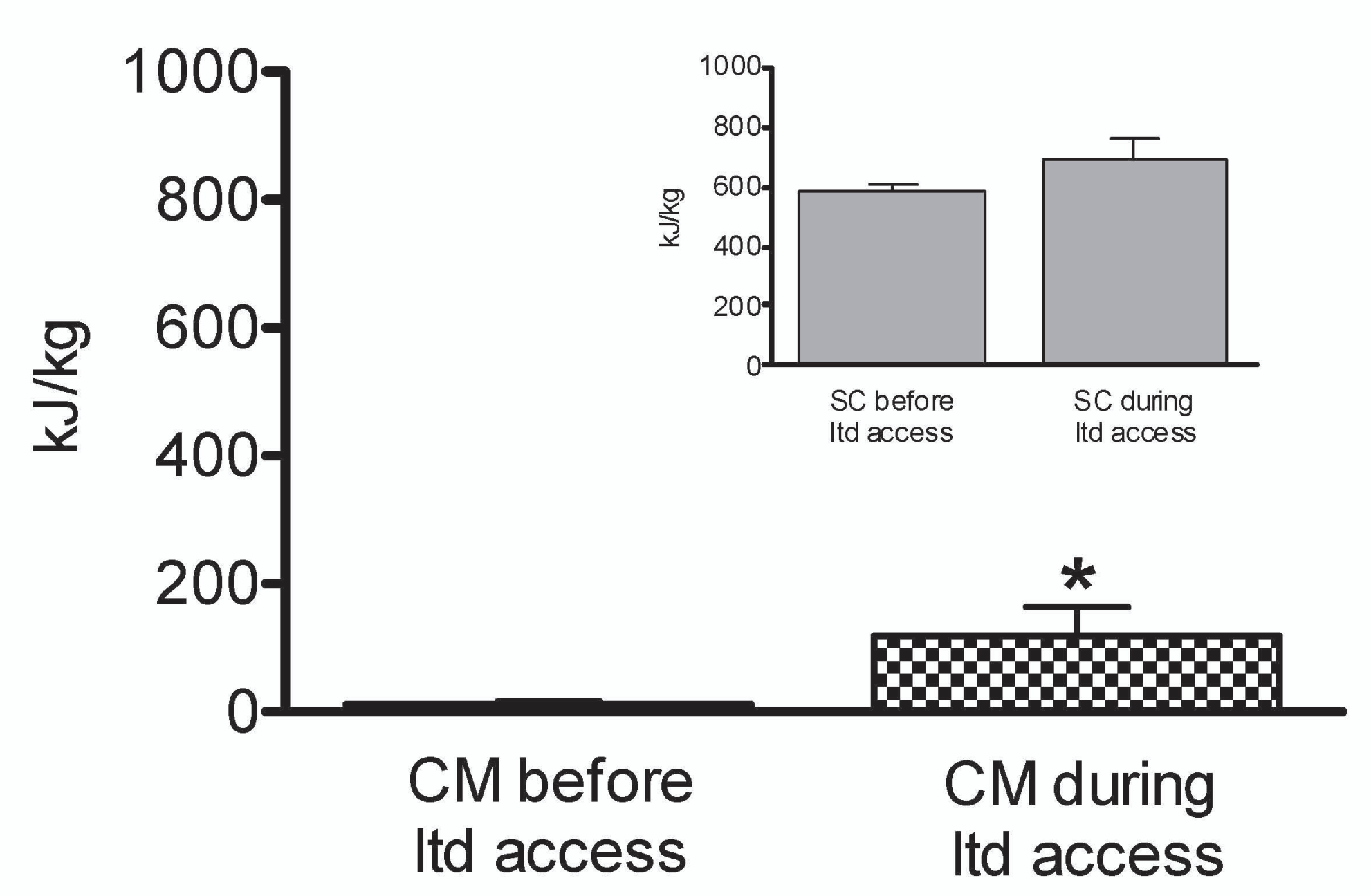

B Eating rate in $1 \mathrm{~h}$ Itd access to HF

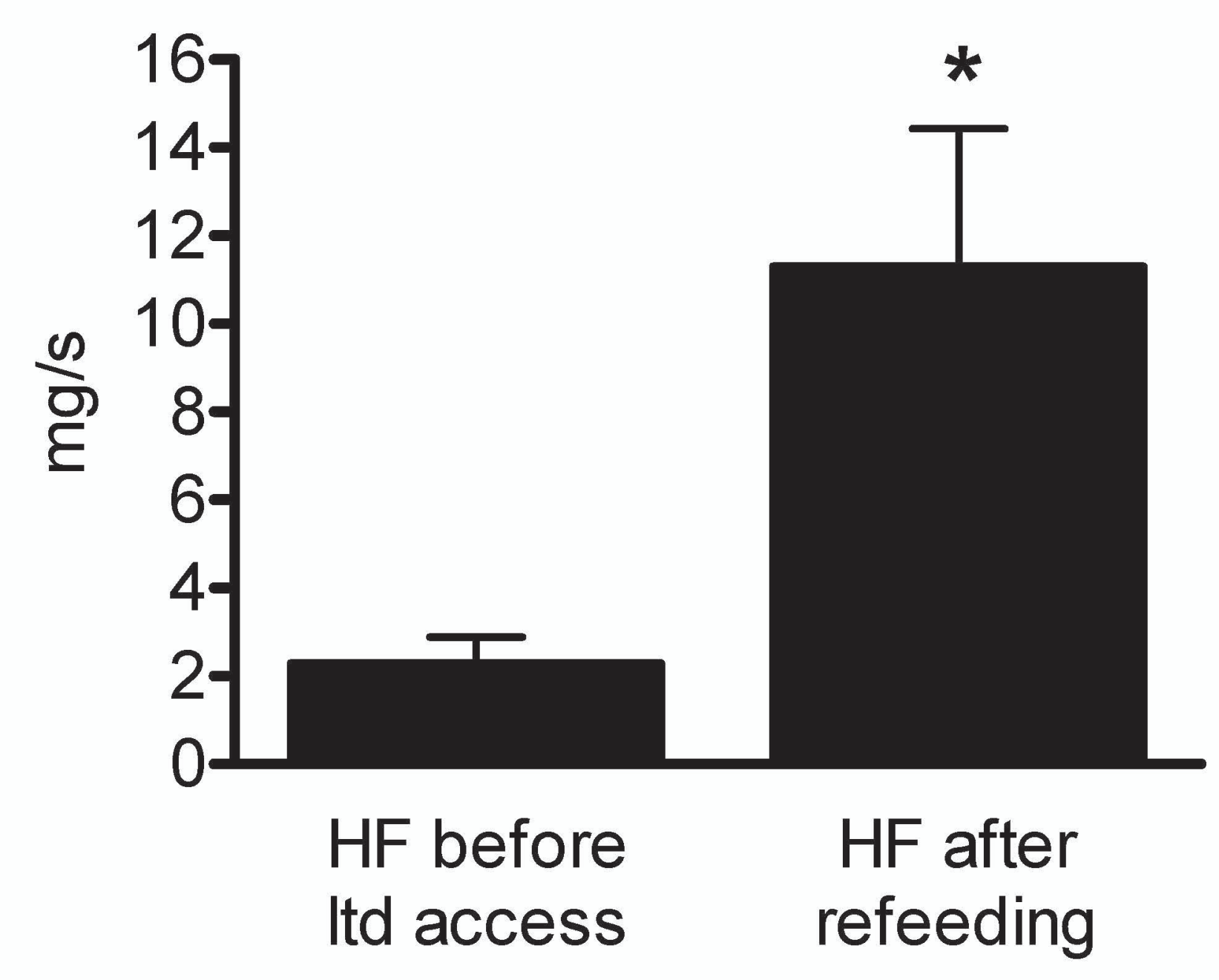

E Eating rate in $1 \mathrm{~h}$ Itd access to CM

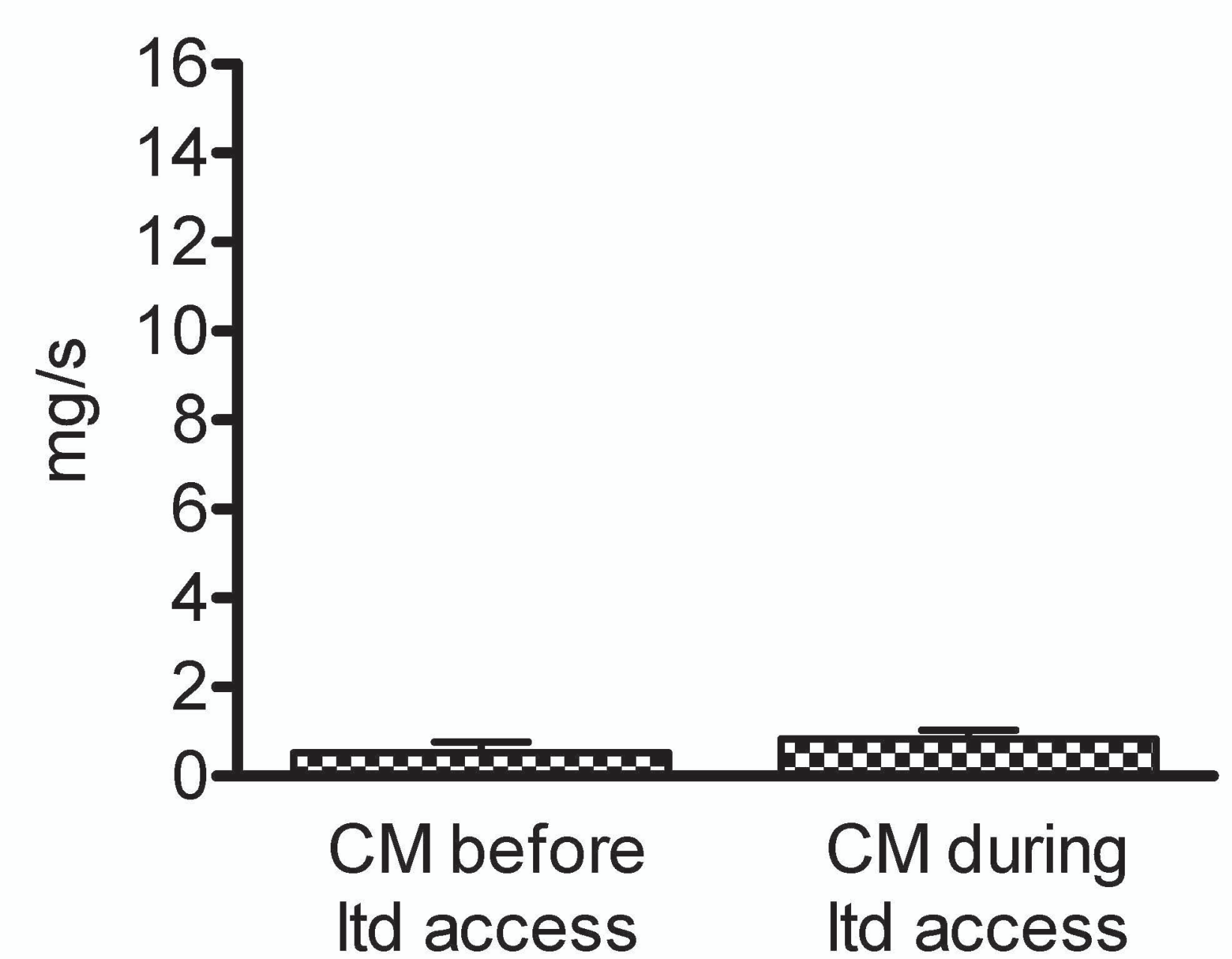

C Average meal size in $1 \mathrm{~h}$ Itd access to HF

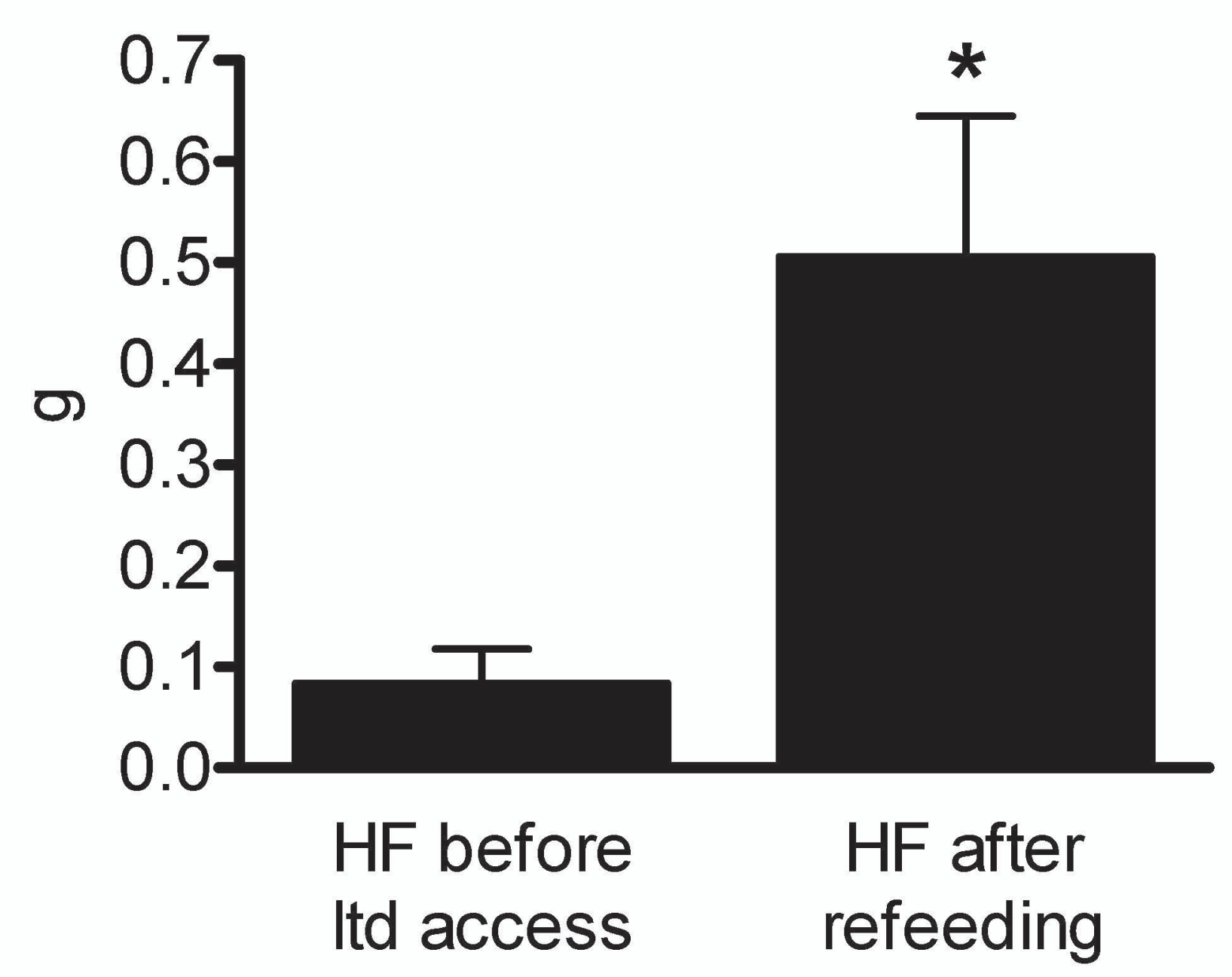

$\mathrm{F}$ Average meal size in $1 \mathrm{~h}$ Itd access to CM

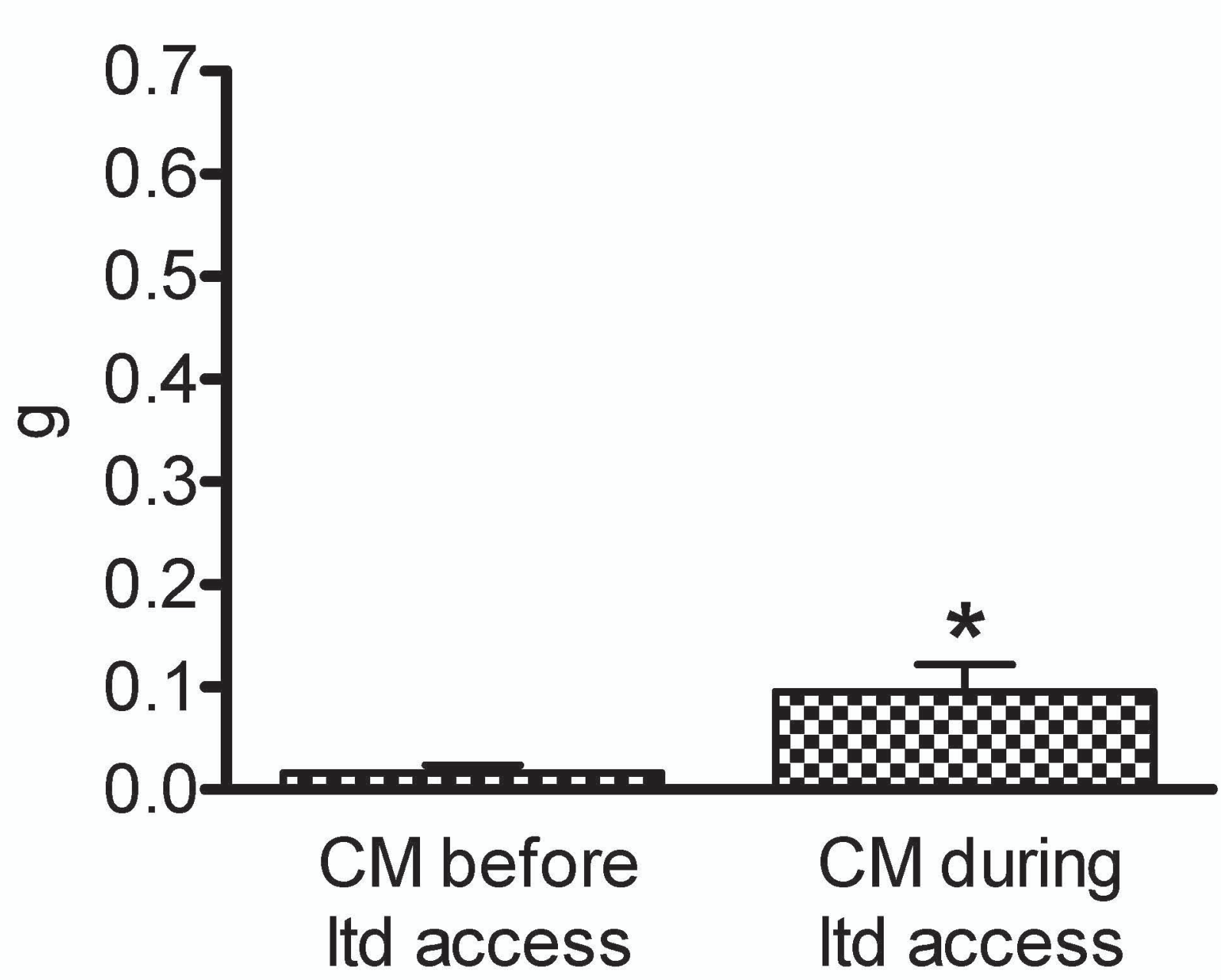


CM vs SC feeder

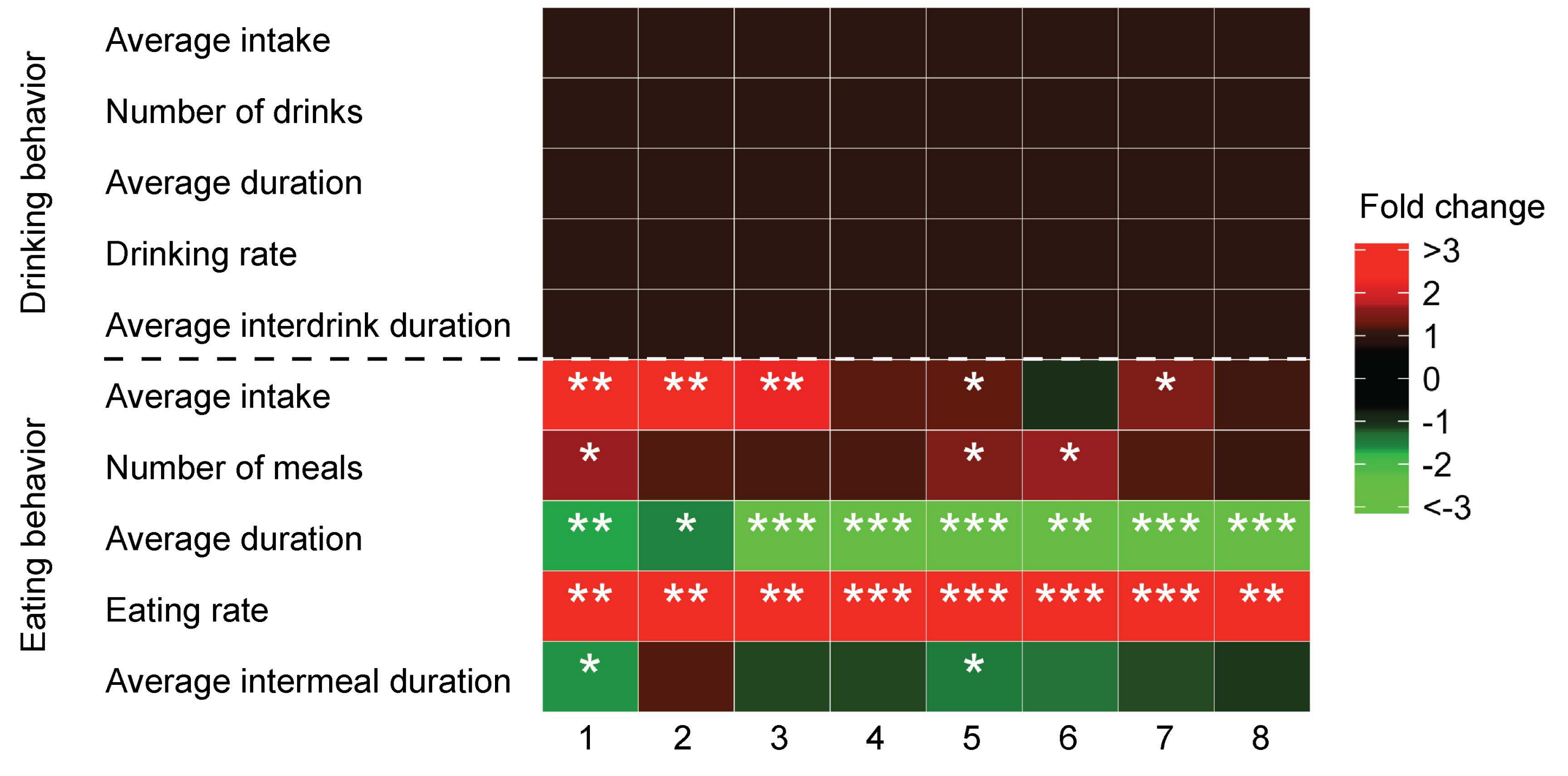

Obesity development phase (weeks) 
$\begin{array}{cc}\text { A } & \begin{array}{c}\text { Average intake during } \\ 30 \mathrm{~min} \text { periods }\end{array}\end{array}$

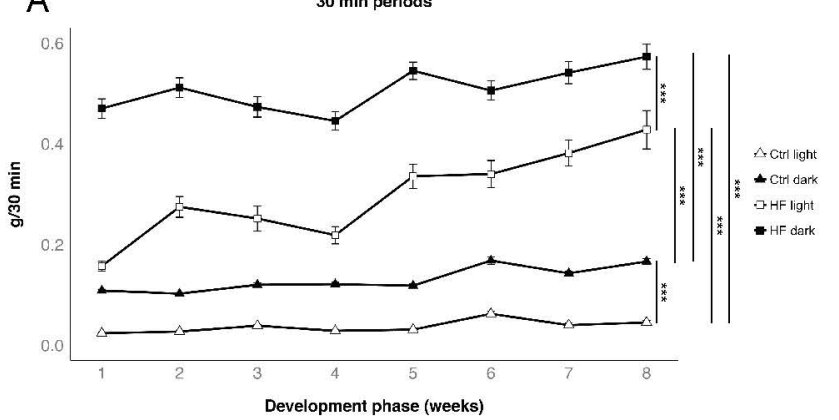

B Mean intake during first $30 \mathrm{~min}$ after cleaning,

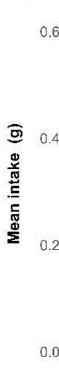

24n before

After cleaning
24h after

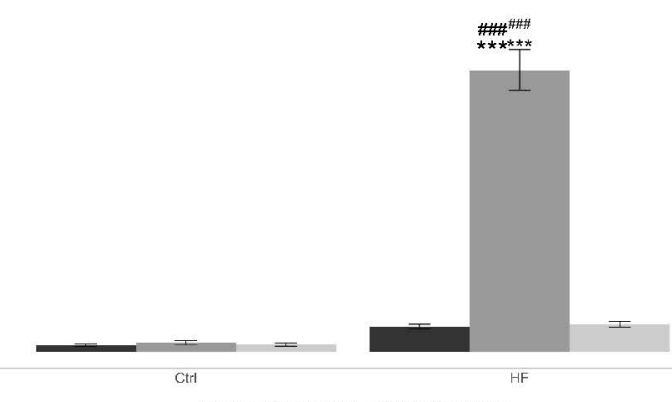

Diet and time respect cleaning starvation 